DIVISION OF THE HUMANITIES AND SOCIAL SCIENCES

CALIFORNIA INSTITUTE OF TECHNOLOGY

PASADENA, CALIFORNIA 91125

THE PRO-COMPETITIVE EFFECT OF CAMPAIGN LIMITS IN NON-MAJORITARIAN ELECTIONS.

Matias laryczower and Andrea Mattozzi

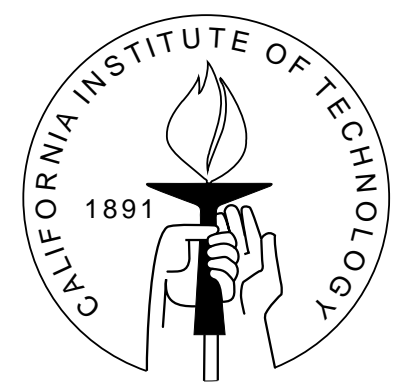

SOCIAL SCIENCE WORKING PAPER 1319

March 2010 


\title{
The Pro-Competitive Effect of Campaign Limits in Non-Majoritarian Elections.
}

\author{
Matias Iaryczower and Andrea Mattozzi
}

\begin{abstract}
We study a model of elections in non-majoritarian systems that captures the link between competition in policies and competition in campaign spending. We argue that the overall competitiveness of the political arena depends both on the endogenous number of parties contesting the election and the endogenous level of campaign spending. These two dimensions are linked together through their combined effect on the total equilibrium level of political rents. We illustrate the key insights of the model through the analysis of two major electoral institutions: campaign spending limits and compulsory voting. In particular, we show that under some conditions spending caps and compulsory voting can be pro-competitive, leading to an increase in the number of parties contesting the elections.
\end{abstract}

JEL classification numbers: D72, D78, C72

Key words: elections, proportional representation, campaign finance, abstention, valence 


\title{
The Pro-Competitive Effect of Campaign Limits in Non-Majoritarian Elections.*
}

\author{
Matias Iaryczower and Andrea Mattozzi ${ }^{\dagger}$
}

\section{Introduction}

Non-majoritarian electoral systems create a link between the degree of ideological differentiation among parties competing for votes, and the incentives of new parties to enter the political arena. Parties that represent policies that are too far apart from one another invite the entry of new competitors, who seek to attract the niche of voters that do not find any of the options available to be particularly appealing. This link is well known in the literature: there is little hope of thinking about representation and ideological differentiation in non-majoritarian elections without letting the number of parties adjust freely in equilibrium. ${ }^{1}$ This, however, is only part of the story.

While parties' ideological positions are surely important in shaping citizens' voting decisions, a paramount ingredient of modern day elections is the campaign competition between parties, and the costs that are associated with it. Parties and candidates spend heavily in electoral campaigns to change voters' impressions about them, and their effort pays off: campaign spending sways votes (Green and Krasno (1988), Kenny and McBurnett (1994), Gerber (1998), Coleman and Manna (2000), Stratmann (2009)), and induces would-be voters to participate in the election (Gerber and Green (2000), Rekkas (2007)).

The key point here is not just that campaign competition is important in modern elections - it is - but that a partial equilibrium analysis of campaign spending is not called for either. Since voters and vote shares are more responsive to differentials in campaign spending between two parties the more similar their policy positions are, a smaller differentiation in policies leads to more intense campaign competition, and then to higher costs of running the campaign in equilibrium. As a result, the effective size of the cake (i.e. total political rents) shrinks, and so does the number of parties that can be supported in equilibrium.

\footnotetext{
* Mattozzi acknowledges financial support from the National Science Foundation, SES-0617901.

$\dagger$ Division of Humanities and Social Sciences, California Institute of Technology, Pasadena, California 91125, USA, emails: miaryc@hss.caltech.edu, andrea@hss.caltech.edu

${ }^{1}$ See Osborne and Slivinski (1996) and Iaryczower and Mattozzi (2009).
} 
In this paper, we introduce a model that captures the link between competition in policies and competition in campaign spending. We illustrate the key insights of the model through the analysis of two major electoral institutions: campaign finance limits (or spending caps) ${ }^{2}$, and compulsory voting. Our analysis brings to light the common effects of these two rather different electoral institutions on the competitiveness of the political arena: under some conditions, both spending caps and compulsory voting can lead to an increase in the number of parties contesting the elections. The key insight of the paper is that the overall competitiveness of the political arena depends both on the endogenous number of parties contesting the election and the endogenous level of campaign competition. Our model shows that these two dimensions are linked together through their combined effect on the total equilibrium level of political rents.

In the first part of the paper we analyze the effect of spending caps on equilibrium outcomes assuming that voting is compulsory. We show that increasing the spending cap leads (eventually) to a smaller number of parties, reducing the set of alternatives available to voters. In fact, increasing the spending cap can eliminate competition altogether. Thus, while relaxing the spending cap can increase the competitiveness of the campaign for the parties that participate in the election, it can also reduce the number of parties that enter the competition, and thus also competition in the ideological dimension.

Our results might suggest that if voters become more responsive to campaigning thus inducing parties to engage in a more intense campaign competition - the equilibrium number of parties should be smaller as well. We show that this is not necessarily the case. In particular, we provide sufficient conditions for equilibrium that are determined by "supply-side" factors only; i.e., the variable cost of campaigning at the spending cap, or the fixed cost of entry into the electoral competition. The responsiveness of voters to campaigning plays no role in defining the number of parties that can be competing for votes in equilibrium.

In the second part of the paper, we allow citizens to abstain from voting in the context of a standard pivotal-voter theory of turnout. We focus on how ideological polarization and campaign competition affect individuals' decisions of whether to vote at all or not, and on how the possibility of abstention affects parties' incentives to spend in campaigning. We first show that in an equilibrium with two parties, an increase in polarization increases turnout and decreases campaign spending. We also show, however, that the possibility of abstention has no direct effect on campaign spending. In other words, in a two-party equilibrium, abstention is incentive-neutral. This is partly due to the fact that individuals who choose to abstain must be locally centrist: they must have ideal policies around that of the individual who is indifferent between the two parties.

We then show that these conclusions do not generally extend to multiparty equilibria. In particular, the two-party logic breaks down in the multiparty setting because absten-

\footnotetext{
${ }^{2}$ As Prat (2002) puts it, "In principle one may restrict [campaign contributions] or [campaign spending], or both. The U.S. Supreme Court has ruled that limits on spending are unconstitutional because they restrict the right to free speech. In contrast, limits on spending are in place in most European countries."
} 
tion has a global effect on representation. With two parties, abstention only transfers power from one party to the other. With more than two parties, the decision of whether to abstain or not affects the vote share of all parties competing for votes, independently of their position in the ideological spectrum. Since abstention has global effects, the entire profile of ideological positions represented by parties running for office becomes relevant. As a result of this, with multiple parties voters' abstention becomes generically non neutral for campaign incentives. We illustrate this in the context of an equilibrium with three parties. We show that in any such equilibrium, at least one party spends more in campaigning when voting is voluntary and costly than when it is compulsory or costless. Furthermore, we show that if the polarization and asymmetry of parties' ideological positions are sufficiently large, then all parties spend more resources in campaigning when voting is voluntary and costly than when it is costless or compulsory.

Our results highlight the importance of looking at total political rents - and their effect on the competitiveness of elections - when evaluating institutions designed to regulate political competition.

This paper builds on a large literature, touching on each of the components of the model. A number of influential papers study elections in majoritarian and proportional systems. Myerson (1993) and Lizzeri and Persico (2001) focus on how the nature of electoral competition affects promises of redistribution made by candidates in the election. Austen-Smith and Banks (1988), Baron and Diermeier (2001), Austen-Smith (2000), Persson, Roland, and Tabellini (2003), and Persson, Tabellini, and Trebbi (2006) consider models of elections and legislative outcomes in proportional representation systems were rational voters anticipate the effect of their vote on the bargaining game between parties in the elected legislature. They do this in the context of a fixed number of parties (and without introducing campaign competition). Palfrey (1984, 1989), Feddersen, Sened, and Wright (1990), Feddersen (1992), Osborne and Slivinski (1996), Besley and Coate (1997), and Iaryczower and Mattozzi (2009) introduce entry in elections. With few exceptions (Osborne and Slivinski (1996), Iaryczower and Mattozzi (2009)), these papers work in the context of plurality elections. ${ }^{3}$

A second strand of literature deals with the campaign competition dimension. In our paper we formalize campaign competition as differentiation in a common value dimension. This builds on the large literature that, following Stokes (1963)'s original critique to the Downsian model, incorporates competition in valence issues (typically within majoritarian electoral systems, and two exogenously given parties). See Groseclose (2001), Aragones and Palfrey (2002), and Berndardt, Camara, and Squintani (2009) for models where one party has an exogenous valence advantage. For models of endogenous valence see Carrillo and Castanheira (2006), Meirowitz (2007), Ashworth and Bueno de Mesquita (2007), Eyster and Kittsteiner (2007), Callander (2008), and Herrera, Levine, and Martinelli (2008).

\footnotetext{
${ }^{3}$ For models of differentiation and entry in industrial organization, see ?, ?, and ?.
} 
Finally, for formal models of abstention with costly voting, see Ledyard (1984), Palfrey and Rosenthal (1983, 1985), Börgers (2004), Krasa and Polborn (2009), and Taylor and Yildirim (2009) (see also Krishna and Morgan (2009) in a common value setting). One common feature of this literature is that only two policy alternatives/parties are considered. Furthermore, it abstracts from the effect of campaign spending on individual turnout decisions. ${ }^{4}$

The rest of the paper is organized as follows. Section 2 presents the model. In Section 3 we characterize equilibria of the model with a focus on the analysis of spending caps. We do this in the context of costless/compulsory voting. In Section 4 we introduce costly voting and the possibility of abstention.

\section{The Model}

There are three stages in the game. In the first stage, a finite set of political parties simultaneously decide whether or not to participate in the election. In the second stage, all parties simultaneously choose a level of campaign spending. In the third stage, a finite set of voters vote.

For given $T$, define the ideology space $X \equiv\{t / T: t=0,1, \ldots, T\} \subset[0,1]$. In any $x \in X$ there is a party who will perfectly represent policy $x$ if elected. ${ }^{5}$ Parties care about the spoils they can appropriate from being in office, and must pay a fixed cost $F$ to participate in the election. We denote the set of parties at the end of the first stage by $\mathcal{K}=\{1, \ldots, K\}$. In the second stage, all parties contesting the election simultaneously choose a level of campaign spending $\theta_{k}$, which cannot exceed a spending cap $L$; i.e., $\theta_{k} \in[0, L]$. Parties can spend $\theta_{k}$ at a cost $C_{v}\left(\theta_{k}\right), C_{v}(\cdot)$ increasing and convex. In the third stage, $N$ fully strategic voters vote in an election, where we think as $N$ being a large finite number. A voter $i$ with ideal point $z^{i} \in X$ ranks parties according to the utility function $u\left(\cdot ; z^{i}\right)$, which assigns to party $k$ with characteristics $\left(\theta_{k}, x_{k}\right)$ the payoff $u\left(\theta_{k}, x_{k} ; z^{i}\right) \equiv 2 \alpha v\left(\theta_{k}\right)-\left(x_{k}-z^{i}\right)^{2}$, with $v$ increasing and concave. The parameter $\alpha$ captures voters' responsiveness to campaigning. Voters' ideal points are uniformly distributed in $X$.

Let $\theta_{\mathcal{K}} \equiv\left\{\theta_{k}\right\}_{k \in \mathcal{K}}$, and $x_{\mathcal{K}} \equiv\left\{x_{k}\right\}_{k \in \mathcal{K}}$ denote the level of campaigning and policy positions of the parties contesting the election. We assume that each party $k$ obtains a share of the total seats in the legislature equal to her share of votes in the election, $s_{k}\left(\theta_{\mathcal{K}}, x_{\mathcal{K}}\right)$, and that the policy outcome is the result of a probabilistic compromise among the parties represented in the legislature, where the likelihood of the policy represented by a party emerging as the policy outcome is increasing in the candidate's vote share,

\footnotetext{
${ }^{4}$ Exceptions are Herrera, Levine, and Martinelli (2008) and Degan (2008). While they both focus on majoritarian elections with two exogenously given parties, the former consider a group turnout model and the latter an uncertain-voter model of turnout.

${ }^{5}$ This assumption captures the fact that commitments to any other alternative policy are not credible in the context of a static model.
} 
or seat share in the assembly. ${ }^{6}$ The expected share of rents appropriated by party $k$, denoted $m_{k}$, is proportional to his vote share in the election. For simplicity, and without any real loss of generality, we assume that $m_{k}\left(\theta_{\mathcal{K}}, x_{\mathcal{K}}\right)=s_{k}\left(\theta_{\mathcal{K}}, x_{\mathcal{K}}\right)$. Normalizing total political rents to one, and letting $C(\cdot) \equiv C_{v}(\cdot)+F$, we can write the expected payoff of a party $k$ contesting the election as

$$
\Pi_{k}\left(\mathcal{K}, x_{\mathcal{K}}, \theta_{\mathcal{K}}\right)=m_{k}\left(\theta_{\mathcal{K}}, x_{\mathcal{K}}\right)-C\left(\theta_{k}\right)
$$

A strategy for party $k$ is a decision of whether or not to participate in the election, and campaign spending $\theta_{k}\left(\mathcal{K}, x_{\mathcal{K}}\right) \geq 0$. A strategy for voter $i$ is a function $\sigma_{i}\left(\mathcal{K}, x_{\mathcal{K}}, \theta_{\mathcal{K}}\right) \in \mathcal{K}$, where $\sigma_{i}\left(\mathcal{K}, x_{\mathcal{K}}, \theta_{\mathcal{K}}\right)=k$ indicates the choice of voting for party $k$, and $\sigma=\left\{\sigma_{1}(\cdot), \ldots, \sigma_{N}(\cdot)\right\}$ denotes a voting strategy profile. An electoral equilibrium is a pure strategy Subgame Perfect Nash Equilibrium of the game of electoral competition, i.e., a strategy profile such that $(i)$ voters cannot obtain a better policy outcome by voting for a different party in any voting game (on and off the equilibrium path), (ii) given the location and campaign decisions of other parties, and given voters' voting strategy, parties cannot increase their expected rents by modifying their campaign levels, (iii) parties contesting the election earn non-negative rents, and (iv) parties not contesting the election prefer not to enter: would earn negative rents in an equilibrium of the continuation game.

Foundations of Campaign Spending. In the model, we directly assume that campaign spending increases a party's vote share without specifying the underlying mechanism. One possible justification for this effect is that campaign spending can reduce voters' uncertainty about candidates' ideological positions. If voters dislike uncertainty over the policies to be implemented by each party, then parties' efforts aimed at informing voters about their policy goals - by publishing and disseminating informative material through TV, newspapers and other media - will be valued by all voters. This idea was first formalized by Austen-Smith (1987), and finds support in the empirical literature. Bartels (1986) shows that voters dislike uncertainty, and that the magnitude of this effect is important. Focusing on data for US legislative elections, Coleman and Manna (2000) show that "Campaign spending increases knowledge of and affect toward the candidates, improves the public's ability to place candidates on ideology and issue scales, and encourages certainty about those placements." Our model is fully consistent with this mechanism, interpreting the common value dimension as reflecting the electorate's uncertainty about the true positions that parties will champion after elections. In particular, this happens generically when voters are policy-risk-averse as in our model given the assumption of a quadratic policy payoff function. In this latter case, we can recover the benchmark model starting from primitives. Suppose then that $U\left(x_{k}, z^{i}\right)=-\beta\left(x_{k}-z^{i}\right)^{2}$, and that the policy $y_{k}$ of candidate $k$ is perceived by voters to be distributed uniformly on $\left[x_{k}-\epsilon(\theta), x_{k}+\epsilon(\theta)\right]$, where $\epsilon(\theta)$ is a decreasing and convex function of campaign spending $\theta$. Then the expected utility of a voter with ideal point $z^{i}$ can be written as $E\left[U\left(x_{k}, z^{i}\right) ; \theta\right]=-\beta\left(x_{k}-z^{i}\right)^{2}+v(\theta)$, where $v(\theta)$ is an increasing and concave function of $\theta$.

\footnotetext{
${ }^{6}$ For a similar approach see, e.g., Grossman and Helpman (1996) and Persico and Sahuguet (2006).
} 
Policy Motivation. In the model we assume that parties are entirely officemotivated; i.e., they do not care about policy outcomes per se, but only as a tool to get votes. ${ }^{7}$ This is a natural assumption in the context of our static model. ${ }^{8}$ However, it is possible to argue that politicians are also policy-motivated. This issue is not a source of concern for any of our results. In fact we can show that if office motivation dominates policy motivation, then all our results are qualitatively unchanged.

Here we provide the main intuition. Policy motivation introduces two effects. The first is on the incentives of parties to participate in the election. When parties have policy preferences, their payoff of participating in the election diminishes the closer the other parties are in the policy space. This is the same effect identified in majoritarian elections by Besley and Coate (1997) and Osborne and Slivinski (1996). But note that in non-majoritarian elections, this "free riding" effect only reinforces a similar mechanism already present even in the absence of policy motivation. In fact in this case, parties' payoffs vary continuously with their vote shares and, as a result, diminish when a party is championing a "crowded" ideological position. (In majoritarian elections, on the other hand, this direct effect cannot occur due to the discontinuity in rents induced by the winner-takes-all nature of the plurality system.) In a nutshell, in non-majoriarian elections, the externality is internalized via vote shares.

Policy motivation also carries a second effect, on parties' incentives to campaign. As we show below, in our model parties compete more intensely in campaigning the closer they are to one another in the ideological space. With policy motivation, this effect is now ameliorated, since as parties get closer to one another, the free-riding effect also reduces the marginal benefit of stealing votes from each other. We can show instead that if office motivation is sufficiently important, the office-induced incentives dominate the policy-induced incentives, and campaign is increasing the closer parties are to one another. As a result, all our analysis is qualitatively unchanged.

\section{The Institutional Determinants of Competition in Short-Run and Long-Run Electoral Equilibria}

We begin our analysis focusing on a short-run horizon in which the number of parties competing in the election is fixed. To differentiate this from our full equilibrium analysis with entry, we call equilibria in this restricted setting short-run electoral equilibria. In a short-run electoral equilibrium, parties competing in the election choose campaign levels that are best responses to one another given their location in the policy space, and

\footnotetext{
${ }^{7}$ As Strom (1990) puts it: "With a slight twist on Downs, we can define a political party as an organization that seeks benefits derived from public office by gaining representation in duly constituted elections." (pg. 574)

${ }^{8}$ See Alesina (1988): "[... in general in a one-shot electoral game the only time-consistent equilibrium is one in which no convergence is possible [...] and the voters rationally expect this outcome." (pg. 796)
} 
must be willing to participate (must earn non-negative rents in equilibrium), but are not concerned about the possible entry of an out-of-equilibrium party. We begin in section 3.1 with the simplest case in which two parties compete in the election. We then move on to the multiple party case in section 3.2. The main result of the section (Theorem 1) is presented in Section 3.3, which deals with long-run electoral equilibria.

\subsection{Short-Run Electoral Equilibrium with Two Parties}

In this section, we establish two classes of results. We start by characterizing equilibria in which parties are unconstrained in campaigning. We then analyze the effect of raising the spending cap, taking voters' characteristics as given.

Consider two candidates 1 and 2 representing policy positions $x_{1}$ and $x_{2}$ with ideological differentiation $\Delta=x_{2}-x_{1}$, and campaign spending $\theta_{1}$ and $\theta_{2}$. Let $\tilde{x}_{12} \in \mathcal{R}$ denote the (unique) value of $x$ such that $u\left(\theta_{1}, x_{1} ; x\right)=u\left(\theta_{2}, x_{2} ; x\right)$, so that $u\left(\theta_{1}, x_{1} ; z^{i}\right)>u\left(\theta_{2}, x_{2} ; z^{i}\right)$ if and only if $z^{i}>\tilde{x}_{12}$

$$
\tilde{x}_{12}=\frac{x_{1}+x_{2}}{2}+\alpha \frac{\left[v\left(\theta_{1}\right)-v\left(\theta_{2}\right)\right]}{\Delta} .
$$

Note that with two parties competing in the election there is no room for strategic voting, and thus all voters vote for their preferred alternative in equilibrium. Hence, as long as $\tilde{x}_{12} \in(0,1)$, candidates vote share mappings are given by $m_{1}(\theta, x)=\tilde{x}_{12}(\theta, x)$, and $m_{2}(\theta, x)=1-\tilde{x}_{12}(\theta, x)$. We first show that when voters are sufficiently ideological and thus relatively unresponsive to campaign spending - it is always possible to support a short-run electoral equilibrium in which parties are not constrained in campaigning. What "sufficiently ideological" means here precisely depends on the cost of campaigning evaluated at the spending cap, $C(L)$. By letting $\Psi(\cdot) \equiv v^{\prime}(\cdot) / C_{v}^{\prime}(\cdot)$, and defining the bound $b \equiv 1 / \Psi(L)$ if $C(L) \leq 1 / 2$ and $b \equiv 1 / \Psi\left(C^{-1}(1 / 2)\right)$ otherwise, we have the following result.

Proposition 1 For any $\alpha \leq b$, there exists a short run electoral equilibrium with two parties unconstrained in campaigning. In any such equilibrium, (i) there is a lower bound on ideological differentiation $\Delta(\alpha)$, which is increasing in $\alpha$, and (ii) campaign competition is neutral for electoral outcomes. In fact

$$
\theta_{1}^{*}=\theta_{2}^{*}=\theta^{*}=\Psi^{-1}(\Delta / \alpha)
$$

The fact that there cannot be a campaign differential between parties is a feature of all equilibria with two parties: while parties' total rents depend on their ideological positions, campaign incentives - i.e., marginal rents - depend only on their ideological 
differentiation. It follows that in a two-party equilibrium both parties must have the same incentives in campaigning.

Note also that even for relatively low responsiveness to campaigning (i.e., $\alpha<b$ ), Proposition 1 identifies a lower bound on ideological differentiation as a necessary condition for parties to be unconstrained in campaigning. This suggests that when instead voters are sufficiently responsive ( $\alpha$ is big enough), no feasible ideological differentiation would induce a soft enough competition. As we show in the next proposition, in such an environment parties always campaign aggressively. However, this does not mean that such intense competition can always be carried out: depending on the total campaign cost at the spending cap, either parties are able to sustain a high level of competition (in which case parties are necessarily constrained by the cap) or, when they are not, the system must lose competition altogether. In other words, when voters are easily swayed by campaign spending, relaxing the spending cap can end up eliminating competition in equilibrium.

Proposition 2 Suppose $\alpha>b$. Then if $C(L) \leq 1 / 2$, there exists a short-run electoral equilibrium with two parties, constrained in campaigning. If instead $C(L)>1 / 2$, then in all pure strategy electoral equilibria a single party runs unopposed, without campaigning.

Two remarks follow. First, note that together with Proposition 1 this result implies that when the spending cap is relatively low, a two-party short-run electoral equilibrium in pure strategies can be sustained independently of the value of $\alpha$. In other words, the conditions constraining the number of parties in equilibrium are entirely determined by "supply side" factors; i.e., the cost of campaigning at the spending cap, $C(L)$. Voters' responsiveness to campaign advertising only affect parties' equilibrium rents.

Second, note that in keeping with the equilibrium notion employed in the paper, the second part of Proposition 2 states that if $C(L)>1 / 2$, then in all electoral equilibria in pure strategies a single party runs unopposed. The result, however, extends to equilibria in mixed strategies as well. Since this is an interesting result in itself, we discuss this briefly below.

Rent Dissipation in Mixed Strategy Equilibria. When $C(L)>1 / 2$ and $\alpha>b$ there cannot be a short-run electoral equilibrium in pure strategies with more than one party. While two-party mixed-strategy equilibria certainly exist in the campaign stage, the more relevant question for our purpose is whether in these equilibria parties will completely dissipate their variable rents (excluding the fixed cost $F$ ). If this were true parties would earn negative total rents. Therefore a short-run electoral equilibrium with two parties does not exist. It turns out that this is exactly what happens when voters' responsiveness to campaign is high enough. To see why this is the case, notice that for sufficiently high $\alpha$, the game of campaign competition can be approximated by an all-pay auction between parties. More precisely, for every $\epsilon>0$ consider the discrete version of the campaigning game where $\theta=\{0, \epsilon, 2 \epsilon, \ldots, L\}$ and, for simplicity, let $C_{v}(L)>1$ 
and $\left(x_{1}+x_{2}\right) / 2=1 / 2$, i.e., the case in which parties ideological position are symmetric and the spending cap is never binding. Simple algebraic manipulation of equation (2) shows that there exists a threshold $\bar{\alpha}(\epsilon) \equiv \Delta / 2 \epsilon$ such that if $\alpha>\bar{\alpha}(\epsilon)$ we can write the expected variable rents of party $k=1,2$ as

$$
\Pi_{k}^{v}= \begin{cases}1-C_{v}\left(\theta_{k}\right) & \text { if } \theta_{k}>\theta_{-k} \\ \frac{1}{2}-C_{v}\left(\theta_{k}\right) & \text { if } \theta_{k}=\theta_{-k} \\ -C_{v}\left(\theta_{k}\right) & \text { if } \theta_{k}<\theta_{-k} .\end{cases}
$$

Since the campaigning stage is a discrete symmetric all-pay auction, we can use the results of Baye, Kovenock, and de Vries (1994) to conclude that there exists a symmetric mixed-strategy equilibrium in which each party puts positive probability on all pure strategies $\theta_{k}$ such that $\theta_{k} \leq C_{v}^{-1}(1)$. As $\epsilon$ approaches zero the equilibrium distributions converge uniformly to the continuous uniform distribution, which is the unique equilibrium of a two-player all-pay auction with a continuous strategy space (Baye, Kovenock, and de Vries (1996)). Further, as $\epsilon$ approaches zero, expected variable rents converge to zero; there is full rent dissipation. As a consequence, for any $\epsilon$ we can find a sufficiently large $\alpha$ such that the variable rents of this mixed-strategy equilibrium of the campaign stage are arbitrarily small and, in particular, smaller than the fixed cost $F$. Hence, restricting attention to the only class of equilibria in the campaign stage that survives when $\epsilon$ approaches zero, a two-party short-run electoral equilibrium does not exist and all equilibria have a single party not investing in campaigning (this equilibrium exists trivially for all values of $(\alpha, L))$.

To sum up, in this section we established two classes of results. First, we characterized equilibria in which parties are unconstrained in campaigning. We showed that for any given spending cap, if voters are sufficiently unresponsive to parties' campaign efforts (i.e., sufficiently ideological), then there exists a short-run equilibrium with two parties unconstrained in campaigning. In any such equilibrium both parties must spend an equal amount on campaigning, and as a result campaign competition is neutral for electoral outcomes. Campaign competition does however affect political rents. In fact we showed that political rents increase - as equilibrium campaign spending decreases - the larger is the ideological differentiation among parties in the election. Second, we considered the effect of raising the spending cap, taking voters' characteristics as given. We showed that when voters are highly responsive to campaigning, raising spending caps can have an anti-competitive effect in equilibrium. This remarkable result is due to the fact that in this situation raising the spending cap increases the cost of campaign competition, and eventually leads to the impossibility of sustaining competition in equilibrium: for high enough $L$, all equilibria (in pure and mixed strategies) have a single party running in the election. 


\subsection{Short-Run Electoral Equilibrium with Multiple Parties}

In this section we extend the previous analysis of short-run electoral equilibria to the case of $K \geq 3$ parties. Considering a multiparty environment introduces some nontrivial theoretical considerations, which we discuss and address immediately below. We then present the main results of this section in Proposition 3 and its corollaries.

The first potential complication in dealing with multiparty equilibria concerns the characterization of the vote share functions. With only two alternatives, there is no room for strategic voting, and thus in equilibrium all voters vote for their preferred party in every subgame. With more than two parties, instead, a voter might conceivably benefit from voting for a party other than her most preferred, if by doing so she reduces the likelihood of ending up with her least preferred policy outcome. Lemma 3 in the Appendix rules out this possibility, and shows that in any voting subgame of any electoral equilibrium, voters vote for their preferred alternative. This result simplifies considerably the characterization of electoral equilibria, assuring uniquely determined, smooth and well behaved vote share functions for all parties on and off the equilibrium path.

The second consideration brought by multiparty competition in non-majoritarian elections concerns the incentives to spend in campaigning, and is substantially more involved. The first point to note is that for any number $K$ of parties competing in the election, small changes in party $k$ 's campaign spending only lead to changes in the distribution of votes between $k$ and its two "effective" competitors, one to each side of the policy spectrum. Given the identity of $k$ 's relevant competitors at a particular campaign spending profile, the marginal impact of $k$ 's campaign spending on vote shares is always local in nature, and therefore well defined. In particular, the marginal benefit of campaign spending increases the larger is voters' responsiveness to campaign spending, the less differentiated $k$ is on average with regards to his effective competitors, and given this, the less symmetric is $k$ 's differentiation with regards to his relevant competitors.

The complication arises because the identity of $k$ 's effective competitors will not necessarily remain fixed at different campaign spending profiles, and in particular it will not always coincide with that of $k$ 's closest neighbors. But since closer parties in the policy space are better substitutes for each other, changes in party $k$ 's campaign spending will have a stronger impact on how voters rank $k$ relative to its closest competitors than to more distant parties in the policy space. As a result, changes in the identity of a party's relevant competitors will lead to non-differentiabilities in the mapping from campaign spending to vote shares and discontinuities in the marginal vote share mapping.

In Proposition 3, however, we show that under some conditions the action identified as optimal by the first order condition will indeed be a best response. In particular, we prove that this is true for all strategy profiles with $K \geq 3$, where all parties representing interior positions $k=2, \ldots, K-1$ choose equal campaign spending; i.e., $\theta_{k}^{*}=\theta^{*}$ for all $k=2, \ldots, K-1$. The intuition for this result is that with symmetry, the discontinuities described above occur at levels of campaigning that are all larger than the optimal 
solution and thus never reached in a best response (the interested reader is referred to Appendix B for a more detailed discussion).

To state this formally, we define a class of electoral equilibria in which all parties contesting the election are located at the same distance to their closest neighbors. We call equilibria of this class location-symmetric (LS) electoral equilibria.

Definition 1 An electoral equilibrium is a location-symmetric (LS) electoral equilibrium if $x_{k+1}-x_{k}=\Delta$ for any $k<K$, and $x_{1}=1-x_{K}$.

The next lemma shows that the non-differentiabilities in the mapping of campaign investment to vote shares discussed above are not relevant in a LS equilibrium.

Lemma 1 Consider a LS equilibrium with $K \geq 3$ parties contesting the election such that $\theta_{k}^{*}<L$ for all $k$. Then parties' equilibrium campaign spending is given by

$$
\theta_{k}^{*}=\Psi^{-1}(\Delta / 2 \alpha) \text { for all } k=2, \ldots, K-1 \text { and } \theta_{1}^{*}=\theta_{K}^{*}=\Psi^{-1}(\Delta / \alpha) \text {. }
$$

Notice that in any LS equilibrium it will always be the case that $\theta_{1}^{*}=\theta_{K}^{*}$ and that $\theta_{k}^{*}=\theta^{*}$ for all $k=2, \ldots, K-1$.

Building on Lemma 1 and Lemma 3 - and letting $\underline{a} \equiv C(L) / 2 \Psi(L)$ and $\bar{a} \equiv$ $C(L) / \Psi(L)$ - the next result extends Propositions 1 and 2 to the case of multiple parties.

Proposition 3 Take an integer $K \geq 3$ as given. If $K \times C(L) \leq 1$ there exists a short-run $L S$ equilibrium with $K$ parties. Moreover, for any $\alpha$ there is a short-run $L S$ equilibrium $\left(x_{1}^{*}(\alpha), \theta_{1}^{*}(\alpha), \Delta^{*}(\alpha), \theta^{*}(\alpha)\right)$ such that

1. If $\alpha \leq \underline{a}$, then all parties are campaign unconstrained, i.e., $\theta_{1}^{*}=\Psi^{-1}\left(\Delta^{*}(\alpha) / \alpha\right)<$ $\theta^{*}(\alpha)=\Psi^{-1}\left(\Delta^{*}(\alpha) / 2 \alpha\right)<L$. Further, for every party $k$ campaign spending is decreasing with ideological differentiation and voters' ideological focus.

2. If $\underline{a} \leq \alpha \leq \bar{a}$, then only parties representing interior ideological positions are constrained by campaign spending caps, i.e., $\theta_{1}^{*}(\alpha)=\Psi^{-1}\left(\Delta^{*}(\alpha) / \alpha\right)<\theta^{*}(\alpha)=L$.

3. If $\alpha \geq \bar{a}$, then all parties are campaign constrained, i.e., $\theta_{1}^{*}(\alpha)=\theta^{*}(\alpha)=L$.

Together with the results of Section 3.1, Proposition 3 implies that for any integer $K \geq 2$ such that $K \times C(L) \leq 1$, there exists a short-run electoral equilibrium with $K$ parties. This result generalizes our previous conclusions, and yields two key implications.

First, note that - differently to a model in which the number of parties is given, in which limiting campaign spending can possibly increase but never reduce parties' rents - here tightening the campaign spending cap can lead to a larger number of parties in 
equilibrium, increasing competitive pressures as a result. By the same logic, relaxing the campaign spending cap leads (eventually) to less parties, reducing the set of alternatives available to voters, and the competitiveness of the election. In fact, as we showed in the previous section for the specific case of two parties, increasing the spending cap can possibly eliminate competition altogether. This is indeed true for any $K$ : we can find an $\alpha$ large enough such that if the spending cap $L$ grows large, the unique short-run equilibrium has a single party not investing in campaigning. ${ }^{9}$ Second, note that the conditions constraining the number of parties in equilibrium are entirely determined by supply side factors: the cost of campaigning at the spending cap, $C(L)$. Instead the responsiveness of voters to campaigning plays no role in defining the number of parties that can be competing for votes in a short-run equilibrium.

The uncoupling of supply and demand factors in the determination of the number of parties does not mean, of course, that "demand-side" factors are irrelevant for equilibrium. Instead, the level of voters' ideological focus impacts the intensity of campaign competition between the given number of parties competing for votes in the election. In particular, for any given spending cap $L$, if voters are sufficiently ideological ( $\alpha$ is low), parties will be unconstrained by spending caps in equilibrium. As voters' ideological focus diminishes, first centrist candidates and eventually all candidates will hit campaign constraints. What makes this possible is that in these equilibria parties earn strictly positive rents from participating in the electoral competition. This allows the system enough flexibility so that the number of parties in the election can be independent of demand-side factors: as voters become more responsive to campaign spending, campaign competition becomes tighter and candidates "compete away" their rents. The campaign finance limits prevent complete rent dissipation. We establish this formally in the next corollary.

Corollary 1 Take $K \geq 3$ as given, and assume $K \times C(L) \leq 1$. Then for any $\alpha$ there is a short-run LS equilibrium $\left(x_{1}^{*}(\alpha), \theta_{1}^{*}(\alpha), \Delta^{*}(\alpha), \theta^{*}(\alpha)\right)$ with associated rents $\left(\Pi_{1}^{*}(\alpha), \Pi^{*}(\alpha)\right)$ for parties representing extreme and interior ideological positions respectively, such that

1. $\Pi_{1}^{*}(\alpha)$ is strictly decreasing for all $\alpha<\bar{a}$, and positive and constant for all $\alpha>\bar{a}$.

2. $\Pi^{*}(\alpha)$ is strictly decreasing for all $\alpha<\underline{a}$, and positive and constant for all $\alpha>\underline{a}$.

It is worth noticing that the mere presence of campaign limits does not prevent complete rent dissipation in equilibrium. Indeed, we can show that there exists an $a^{\prime}$ such that for all $\alpha<a^{\prime}$ there exists a short-run LS equilibrium with at least three parties in which $\theta_{k}^{*}(\alpha)<L$ for all $k$, and all interior parties earn zero rents. This result follows from the second part of Lemma 6 in the appendix.

\footnotetext{
${ }^{9}$ The logic behind this result is very similar to the case of two parties and exploits the property that in any equilibrium of an all-pay auction with any number of players and identical valuations, there is complete rent dissipation (see Theorem 1 of Baye, Kovenock, and de Vries (1996)).
} 


\subsection{Electoral Competition in Long-Run Equilibria}

So far we focused on short-run electoral equilibria, in which incumbent parties cannot be challenged by new parties, even if they represent poor alternatives for a large fraction of voters. In a longer time horizon, however, we expect entry to be a relevant factor in shaping electoral outcomes. In this section, we extend the analysis to long-run electoral equilibria. A strategy profile is a long run electoral equilibrium (or simply an electoral equilibrium) if it is a short-run electoral equilibrium, and in addition parties not participating would obtain negative rents if they chose to enter the electoral competition.

Do our previous short-run results stand in this long-run setting? Remarkably, the answer is an unqualified yes. As in a short-run equilibrium, it is still the case that increasing the spending cap reduces the maximum number of parties that can be contesting the election. In the long-run analysis, in addition, the fixed cost of entry provides a lower bound on the equilibrium number of parties. ${ }^{10}$ Still, as before, there are always conditions for which the responsiveness of voters to campaigning plays no role in defining the number of parties that can be competing for votes in equilibrium. In particular, the uncoupling of supply and demand for the determination of the equilibrium number of parties is still valid in the long-run equilibrium analysis: the equilibrium number of parties is determined entirely by the variable cost of campaigning at the spending cap, $C_{v}(L)$, and the fixed cost of entry $F$. Finally, reducing voters' ideological focus has the effect of increasing the intensity of campaign competition and reducing parties' rents. As in Corollary 1, it is the excess rents in equilibrium which allows the uncoupling of demand and supply side in the determination of the equilibrium number of parties. These results follow from Theorem 1 below.

Theorem 1 Take $K \geq 2$ as given. If $K \times C(L) \leq 1$ and $K \times F \geq 1 / 2$, there exists a long-run electoral equilibrium with $K$ parties. Moreover, there exist thresholds $(\underline{\alpha}, \bar{\alpha})$ and, for any $\alpha$, either a long-run LS equilibrium $\left(x_{1}^{*}(\alpha), \theta_{1}^{*}(\alpha), \Delta^{*}(\alpha), \theta^{*}(\alpha)\right)$ or a two-party electoral equilibrium $\left(x_{1}^{*}(\alpha), \Delta^{*}(\alpha), \theta^{*}(\alpha)\right)$ such that

1. If $\alpha \leq \underline{\alpha}$, then all parties campaign unconstrained, and for every party $k$, campaign spending is decreasing in parties' ideological differentiation. In particular, $\theta_{1}^{*}=$ $\Psi^{-1}\left(\Delta^{*}(\alpha) / \alpha\right)<\theta^{*}(\alpha)=\Psi^{-1}\left(\Delta^{*}(\alpha) / 2 \alpha\right)<L$ for $K>2$, and $\theta^{*}=\Psi^{-1}\left(\Delta^{*}(\alpha) / \alpha\right)$ for $K=2$.

2. If $\alpha \geq \bar{\alpha}$ all parties are campaign constrained.

The main logic driving the results of Theorem 1 is best grasped by considering the special case of two parties unconstrained in campaigning. This is covered in Lemma 2 below.

\footnotetext{
${ }^{10}$ Furthermore, the long-run analysis introduces new bounds on ideological differentiation between parties in equilibrium. We elaborate on this at the end of this section.
} 
Lemma 2 Suppose that $2 \times C(L) \leq 1$ and $2 \times F \geq 1 / 2$. Then for any $\alpha \leq \bar{\alpha}_{2 P}$ there exists a long-run two-party electoral equilibrium $\left(x_{1}^{*}(\alpha), \Delta^{*}(\alpha), \theta^{*}(\alpha)\right)$ such that both parties campaign unconstrained, and campaign spending is decreasing in parties' ideological differentiation. In particular, $\theta^{*}=\Psi^{-1}\left(\Delta^{*}(\alpha) / \alpha\right)<L$.

Proof of Lemma 2. We showed in Proposition 1 that if two parties compete for votes in the election, and (i) voters vote for their preferred party, (ii) both parties are unconstrained in campaign spending and choose

$$
\theta_{k}^{*}=\theta^{*}=\Psi^{-1}\left(\frac{\Delta}{\alpha}\right)
$$

and (iii) parties' ideological differentiation $\Delta \geq \alpha \Psi(L)$ (with $C(L) \leq 1 / 2$ ), then there is a location of the left party $x_{1}$ such that a short-run electoral equilibrium exists. We next show that if conditions (i) and (ii) are satisfied, and parties' ideological differentiation $\Delta \in D^{*}$, where

$$
D^{*} \equiv\left\{\Delta: \max \left\{2 C_{v}(L), 1-2 F, \alpha \Psi(L)\right\} \leq \Delta \leq \min \left\{1-2 C_{v}(L), 2 C(L)\right\}\right\},
$$

then there is a location of the left party $x_{1}$ such that a long-run electoral equilibrium exists. First notice that for $D^{*}$ to be nonempty it is sufficient that

$$
\alpha \leq \min \left\{2 \frac{C(L)}{\Psi(L)}, \frac{1-2 C_{v}(L)}{\Psi(L)}\right\} \equiv \bar{\alpha}_{2 P},
$$

and $C(L)=C_{v}(L)+F \leq 1 / 2$ and $F \geq 1 / 4$ (which in turn implies $\left.C_{v}(L) \leq F\right)$. Hence, if $2 \times C(L) \leq 1,2 \times F \geq 1 / 2$, and $\alpha \leq \bar{\alpha}_{2 P}$ a long-run two-party electoral equilibrium exists. We now show why these conditions are sufficient.

Let $\Delta=\alpha \Psi(L)(1+\varepsilon)$ for $\varepsilon>0$. First we show that entry in $\left(x_{1}, x_{2}\right)$ is not profitable. Suppose that $j$ enters at $x_{j} \in\left(x_{1}, x_{2}\right)$, and consider the following continuation: $\hat{\theta}_{1}=$ $\hat{\theta}_{2}=\hat{\theta}_{j}=1$. Letting $\delta_{j}^{r} \equiv\left(x_{2}-x_{j}\right) / \Delta$, we have that the necessary first order conditions (FOC) for a maximum for $k=1$ and $k=2$ are

$$
\frac{\alpha}{\left(1-\delta_{j}^{r}\right) \Delta} v^{\prime}(L) \geq C_{v}^{\prime}(L) \text { and } \frac{\alpha}{\delta_{j}^{r} \Delta} v^{\prime}(L) \geq C_{v}^{\prime}(L),
$$

while the FOC for $j$ is $\alpha v^{\prime}(L) \geq \delta_{j}^{r}\left(1-\delta_{j}^{r}\right) \Delta C_{v}^{\prime}(L)$ which is implied by the previous inequalities. These conditions are satisfied if and only if

$$
\max \left\{\delta_{j}^{r} \Delta,\left(1-\delta_{j}^{r}\right) \Delta\right\} \leq \alpha \Psi(L) .
$$

Suppose first that $\delta_{j}^{r} \leq 1 / 2$. Then (5) is $\left(1-\delta_{j}^{r}\right) \Delta \leq \alpha \Psi(L)$, or substituting $\Delta=$ $\alpha \Psi(L)(1+\varepsilon), \delta_{j}^{r} \geq \varepsilon /(1+\varepsilon)$. When instead $\delta_{j}^{r} \geq 1 / 2$, then (5) is $\delta_{j}^{r} \Delta \leq \alpha \Psi(L)$, or substituting $\Delta=\alpha \Psi(L)(1+\varepsilon), \delta_{j}^{r} \leq 1 /(1+\varepsilon)$. Thus

$$
\frac{\varepsilon}{1+\varepsilon} \leq \delta_{j}^{r} \leq \frac{1}{1+\varepsilon} .
$$


Note that since $\varepsilon>0$, the interval defined in (6) is strictly included in $(0,1)$. Thus for relatively centrist entrants, $\hat{\theta}_{1}=\hat{\theta}_{2}=\hat{\theta}_{j}=L$ is a joint best response provided that the incumbent parties choose not to quit campaigning. To insure that this is the case, it is enough to consider the case of $\delta_{j}^{r}$ approaching either 0 or $1 .{ }^{11}$ Hence, a necessary and sufficient condition for party 1 not to quit campaigning upon entry is $x_{1} \geq C_{v}(L)$. The case of $\delta_{j}^{r}$ reaching its upper bound is similar and yields $1-x_{2} \geq C_{v}(L)$ as a necessary and sufficient condition for party 2 to prefer not to quit campaigning. Since $x_{1}+\Delta+1-x_{2}=1$, these conditions can be satisfied if and only if $\Delta \leq 1-2 C_{v}(L)$.

Now, given that $\hat{\theta}_{1}=\hat{\theta}_{2}=\hat{\theta}_{j}=L$ we have that $\Pi_{j}\left(\hat{\theta}_{j}\right)=\Delta / 2-C(L)<0$ if and only if $\Delta<2 C(L)$. Next, consider entry such that $\delta_{j}^{r}>1 /(1+\varepsilon)$. Here $j$ enters relatively close to $k=1$, and the strategy profile in the continuation game in which all three parties choose $L$ cannot be an equilibrium. Consider instead $\hat{\theta}_{2} \in(0,1)$, and $\hat{\theta}_{1}=\hat{\theta}_{j}=L$. The necessary first order condition for $k=2$ is

$$
\hat{\theta}_{2}=\Psi^{-1}\left(\frac{\delta_{j}^{r} \Delta}{\alpha}\right)=\Psi^{-1}\left(\delta_{j}^{r} \Psi(L)(1+\varepsilon)\right)<L,
$$

where the second equality follows from $\Delta=\alpha \Psi(L)(1+\varepsilon)$, and the inequality follows from the fact that $\delta_{j}^{r}>1 /(1+\varepsilon)$, and that $\Psi(\cdot)$ is decreasing. The FOC for $j$ is not relevant. The FOC for $k=1$ is $\left(1-\delta_{j}^{r}\right) \Delta \leq \alpha \Psi(L)$, which is implied by $\delta_{j}^{r}>1 /(1+\varepsilon)$. We now need to show that

$$
\Pi_{j}\left(\hat{\theta}_{j}\right)=\tilde{x}_{j 2}\left(L, \hat{\theta}_{2}\right)-\frac{x_{1}+x_{j}}{2}-C_{v}(L)-F<0 .
$$

Now if $\tilde{x}_{1 j}$ were fixed, $j$ would be better off choosing $\tilde{\theta}_{j}=\hat{\theta}_{2}$ (as in the case of a twoparties equilibrium). But then $\hat{\Pi}_{j}<\Delta / 2-C_{v}(L)-F<0$ from $\Delta<2\left(C_{v}(L)+F\right)$. As before, we need to make sure that the incumbent parties choose not to quit campaigning in the continuation game. However, from the previous discussion we know that there exist parameters values for which incumbent parties will not quit campaigning as long as $\Delta \leq 1-2 C_{v}(L)$.

To deter entry in $\left[0, x_{1}\right)$ and $\left(x_{2}, 1\right]$ it is sufficient that $(1) \Delta \geq 2 C_{v}(L)$, and $(2)$ $\Delta \geq 1-2 F$. Condition (1) guarantees that the incumbents are not quitting campaigning upon entry. Condition (2) is a sufficient condition for the existence of $x_{1}$, and $x_{2}$ such that $\max \left\{x_{1}, 1-x_{2}\right\} \leq F$. The latter inequality is clearly enough to guarantee no entry of an extreme party, and since $x_{1}+\Delta+1-x_{2}=1$, it can be satisfied if and only if $\Delta \geq 1-2 F$.

We conclude this section with two observations. First, while Theorem 1 refers to the case of $K \geq 2$, extending the result to a single party is straightforward. First, it is

\footnotetext{
${ }^{11}$ The reason for this is that when $\delta_{j}^{r}$ reaches say its lower bound (i.e., when entry occurs in a right neighborhood of $x_{1}$ ), than necessarily $\hat{\theta}_{1}=\hat{\theta}_{j}=L$ is optimal. Hence, the incumbent rents approaches $x_{1}$, which must be larger than $C_{v}(L)$ in order for party 1 to prefer not to quit campaigning. When instead $\delta_{j}^{r}$ is strictly bigger than zero, either $\hat{\theta}_{1}=L$ is still optimal and necessarily $\left.\Pi_{1}\left(\hat{\theta}_{1}\right)\right|_{\hat{\theta}_{1}=L} \geq x_{1}$, or if $\hat{\theta}_{1}<L$ is optimal then it must be that $\left.\Pi_{1}\left(\hat{\theta}_{1}\right)\right|_{\hat{\theta}_{1}<L}>\left.\Pi_{1}\left(\hat{\theta}_{1}\right)\right|_{\hat{\theta}_{1}=L} \geq x_{1}$.
} 
immediate to verify that there always exists a short-run electoral equilibrium with one party running uncontested. Furthermore, in the case of a single party, the condition $K \times F \geq 1 / 2$ becomes $F \geq 1 / 2$. Hence, if the incumbent is located at the preferred position of the median voter, $x=1 / 2$, the potential entry of a challenger is always deterred. To see why this must be the case, note that the continuation game after entry of a challenger must be a two-party equilibrium, and therefore campaign competition will be neutral for electoral outcomes in that continuation game. Since the incumbent is located at the median, it follows that the challenger's vote share will always be smaller than $1 / 2$. But then $F \geq 1 / 2$ implies that the entrant would earn negative total rents. ${ }^{12}$

Second, when voters are sufficiently responsive to campaign spending, some of the sufficient conditions we used in the proof of Theorem 1 become necessary. We can then show that as $L$ increases the set of long-run two-party electoral equilibria converges to the equilibrium that maximizes ideological representation, i.e., the location of parties that minimizes the total distance between voters' ideological preferences and parties' positions. This finding echoes a well-know result in industrial organization since $x_{1}=1-x_{2}=1 / 4$ is the socially optimal location of two competing shops in the unit interval; i.e., the one that minimizes buyers' transportation costs (see Hotelling (1929)).

Proposition 4 Suppose that the conditions of Theorem 1 are satisfied for $K=2$. Then there exists an $\alpha^{* *}$ such that for all $\alpha>\alpha^{* *}$, as $L$ increases the set of long-run twoparty electoral equilibria converges to a single equilibrium $\left(x_{1}^{* *}(\alpha), \Delta^{* *}(\alpha), \theta^{* *}(\alpha)\right)$. In this equilibrium, parties are positioned in the unique symmetric configuration that maximizes voters' ideological representation.

To sum up, in this section we uncovered an important interaction between campaign spending and the competitiveness of the political arena. In particular, we argued that the overall competitiveness of the political sector depends both on the endogenous number of parties contesting the election and the endogenous level of campaign competition. Our model shows that these two dimensions are linked together through their combined effect on the total equilibrium level of rents. Campaign finance regulation crucially affects this mechanism. We have shown that regulating campaign spending may avoid a perverse anti-competitive effect, that is due to the fact that increasing spending caps heightens campaign competition, reduces political rents, and as a result reduces the number of parties contesting the election.

In the next section, we study the effects of a rather different policy - compulsory voting - on the competitiveness of the political sector. It turns out that under some conditions, compulsory voting and campaign spending limits have a similar effect, and can both lead to an increased competition in the policy dimension.

\footnotetext{
${ }^{12}$ Note that since the incumbent obtains more than half of all votes, and $C_{v}(\theta) \leq 1 / 2$ for all $\theta \leq L$ by hypothesis, the incumbent has an incentive to campaign in any continuation game following entry.
} 


\section{Turnout and Compulsory Voting}

When voting is costless abstention is dominated, and a voter's strategy is restricted to choosing which of the parties participating in the election they will vote for. In this section, we consider the case in which voting is costly. In this case, a fraction of the voters might choose to abstain in equilibrium if they are free to do so, and full participation will generally require a policy of compulsory voting. Our goal is to compare equilibrium outcomes under compulsory and voluntary voting. We consider two related issues. First, we focus on how the number of parties competing in the election, the policy positions they represent, and their level of campaign spending affect which and how many voters abstain. Second, we study how the possibility of abstention affects the intensity of campaign competition in equilibrium.

For our present purposes, it is enough to focus on short-run electoral equilibria in which parties are unconstrained in campaign spending. We begin with the two-party case. We show that in this context only centrist voters abstain. Furthermore, an increase in polarization increases turnout and decreases campaign spending. The possibility of abstention, however, has no direct effect on campaign spending. We then show that these conclusions do not generally extend to multiparty equilibria. In particular, we show that with more than two parties, abstention does have a direct effect on campaign spending.

Consider a modified version of the model of Section 2 in which $N$ voters choose not only which party to vote for if they vote, but also if they want to vote at all (at cost $\tau>0$ ) or abstain. We begin by analyzing symmetric electoral equilibria in which two parties $k=1,2$ compete for votes in the election. We can thus write $x_{1}=1 / 2-\Delta / 2$ and $x_{2}=1 / 2+\Delta / 2$ for $\Delta \in(0,1)$. We look for an equilibrium in which abstainers have ideological positions in an interval $\left(\tilde{x}_{12}^{\ell}, \tilde{x}_{12}^{r}\right)$ around that of the critical individual $\tilde{x}_{12}$ who is indifferent between the two parties (as defined in (2)). We then show in the proof of Proposition 5 that all electoral equilibria with two parties in which some voters abstain must have this property.

Note that $\tilde{x}_{12}^{\ell}$ (similarly, $\tilde{x}_{12}^{r}$ ) is given by the position of the voter who is indifferent between voting for party 1 (party 2 ) and abstaining. Thus $\left(\tilde{x}_{12}^{\ell}, \tilde{x}_{12}^{r}\right)$ solves the system of equations

$$
\left\{\begin{array}{c}
\frac{\left(1-\tilde{x}_{12}^{r}\right)\left(u\left(\theta_{1}, x_{1} ; \tilde{x}_{12}^{\ell}\right)-u\left(\theta_{2}, x_{2} ; \tilde{x}_{12}^{\ell}\right)\right)}{N\left(1-\tilde{x}_{12}^{r}+\tilde{x}_{12}^{\ell}\right)\left(1-\tilde{x}_{12}^{r}+\tilde{x}_{12}^{\ell}-\frac{1}{N}\right)}=\tau \\
\frac{\tilde{x}_{12}^{\ell}\left(u\left(\theta_{2}, x_{2} ; \tilde{x}_{12}^{r}\right)-u\left(\theta_{1}, x_{1} ; \tilde{x}_{1}^{r}\right)\right)}{N\left(1-\tilde{x}_{12}^{r}+\tilde{x}_{12}^{\ell}\right)\left(1-\tilde{x}_{12}^{r}+\tilde{x}_{12}^{\ell}-\frac{1}{N}\right)}=\tau,
\end{array}\right.
$$

which in turn can be written as

$$
\left\{\begin{array}{c}
\tilde{x}_{12}^{\ell}=\tilde{x}_{12} t \\
\tilde{x}_{12}^{r}=1-\left(1-\tilde{x}_{12}\right) t,
\end{array}\right.
$$

where $t$ is the total voter turnout

$$
t=\frac{2 \Delta\left(1-\tilde{x}_{12}\right) \tilde{x}_{12}+\tau}{2 \Delta\left(1-\tilde{x}_{12}\right) \tilde{x}_{12}+N \tau}
$$


These results have important implications for the characterization of two-party equilibria with costly voluntary voting. Consider a sequence of games with increasing population sizes. For there to be an equilibrium with abstention even in the limit as $N \rightarrow \infty$, it must be that $\lim _{N \rightarrow \infty} \lim _{\tau \rightarrow 0} N \tau=A>0$. Then

$$
\tilde{t} \equiv \lim _{N \rightarrow \infty} \lim _{\tau \rightarrow 0} t=\frac{1}{1+\frac{A}{2 \Delta\left(1-\tilde{x}_{12}\right) \tilde{x}_{12}}}=\frac{1}{1+\frac{A}{\frac{1}{2} \Delta-2\left[\alpha\left(v\left(\theta_{1}\right)-v\left(\theta_{2}\right)\right)\right]^{2}}}
$$

Note that voter turnout is an increasing function of polarization $\Delta$, and that turnout vanishes as $\Delta$ approaches 0 . This implies that the possibility of abstention imposes a lower bound on ideological differentiation between parties even in the absence of the threat of entry. Second, note that voter turnout is a decreasing function of (voters' valuation of) the campaign differential between parties, $\alpha\left|v\left(\theta_{1}\right)-v\left(\theta_{2}\right)\right|$. It follows from this that whenever parties are matching each other campaign spending levels, an increase in campaign spending by party $j$ will reduce total voter turnout.

The previous analysis might suggest that the possibility of abstention alters parties' incentives to engage in campaign competition. This, however, is not the case. Note that from (7), given candidates positions $\Delta$ and campaign spending levels $\left(\theta_{1}, \theta_{2}\right)$, the vote share of party 1 is $m_{1}\left(\Delta, \theta_{1}, \theta_{2}\right)=\tilde{x}_{12}^{\ell} / t=\tilde{x}_{12}$, as in the compulsory voting model.

To see why voluntary voting does not affect campaign competition with two parties, it is useful to compare the role of campaigning in the benchmark model with costless/compulsory voting, with its role in the extended model with costly voluntary voting. In the benchmark model, campaign spending by one party has the effect of directly "stealing" votes from the opposition. With abstention, however, campaign spending by party 1 encourages more of its own supporters to vote and - by also making it more attractive to party 2 supporters - reduces the turnout of the competitor's supporters (i.e., increases $\tilde{x}_{12}^{\ell}$ and $\left.\tilde{x}_{12}^{r}\right)$. Simple inspection of (7) shows that in any two-party equilibrium the mass of individuals supporting one party and showing up to the poll is linear in total turnout. Therefore the two effects exactly offset each other: campaign spending attracts less party 1 supporters than in the compulsory voting model, but it reduces total turnout proportionally at party 2's expense. Overall, the change in vote share exactly adds up to the vote stealing effect of the compulsory voting model. We conclude that in an equilibrium with two parties, abstention is incentive-neutral.

Putting everything together, it follows that while in equilibrium turnout $t^{*}=(\Delta+$ $2 \tau) /(\Delta+2 N \tau)$ is increasing in polarization $\Delta-$ so that when polarization increases, campaign spending decreases and turnout increases - the intensity of campaign competition has no direct effect on voter turnout. Without any other alternatives entering into voters' calculations, individuals who choose to abstain must be locally centrist: must have ideal policies around that of the individual who is indifferent between the two parties.

Note that the the fact that parties are located symmetrically around the median does not play any role in the argument. These conclusions hold in all electoral equilibria with two parties. Proposition 5 state these results formally. 
Proposition 5 In any electoral equilibrium with two parties, abstainers have ideal policies contained in an interval around the position of the voter who is indifferent between parties. Furthermore, turnout is increasing in polarization but unaffected by the intensity of campaign competition: abstention is incentive-neutral.

With more than two parties competing for votes, the logic behind Proposition 5 breaks down. The reason is that with multiple parties, abstention has a global effect on representation. With more parties, the decision of whether to abstain or not involves two considerations. One is local in nature: who to vote for if one votes. This only affects the two relevant options for any voter, say $k$ and $k+1$. The second consideration has global consequences. When the voter votes for $k$ instead of not voting at all, she diminishes the vote share of all parties competing for votes, both near or far in the ideological spectrum.

To illustrate the working of these channels in equilibria with multiple parties it is enough to consider equilibria with three parties. Now, since abstention has global effects, the entire profile of ideological positions represented by parties contesting the election becomes relevant. As a result, focusing exclusively on LS equilibria entails a significant loss of generality. We will therefore distinguish between symmetric and asymmetric equilibria in this context. In Proposition 6 we show that when the configuration of parties is symmetric, abstainers are globally centrists: not indifferent between parties $j$ and $k$, or $k$ and $\ell$, but instead around the policy position represented by the centrist party. In an equilibrium of this kind, all abstainers - and in fact all supporters of the centrist party - are indifferent between voting for their preferred candidate and abstaining (the equilibrium turnout rate attains this indifference). In an asymmetric equilibrium instead, abstention is local, but can only occur on one side of the median: the ideologies of abstainers are represented either by an interval around $\tilde{x}_{12}$ or by an interval around $\tilde{x}_{23}$.

Proposition 6 Consider an electoral equilibrium with three parties. If the equilibrium is location-symmetric, abstainers have ideal policies contained in an interval around the position of the centrist party. If instead the equilibrium is asymmetric, abstainers' ideal policies are either contained in an interval around $\tilde{x}_{12}$ or in an interval around $\tilde{x}_{23}$.

To get some intuition for how these results come about, consider first a highly asymmetric equilibrium, with $\left|x_{3}-x_{2}\right|<<\left|x_{1}-x_{2}\right|$. Note that as $\left|x_{2}-x_{3}\right| \rightarrow 0$, voters effectively face a choice between two alternatives, and we have established before that in this case abstainers are in an interval around the individual who is indifferent between the two parties, $\tilde{x}_{12}$. The highly asymmetric equilibrium with three parties will still be different than the two party equilibrium because parties 2 and 3 spend more in campaigning than what a single party 2 would spend, but this higher spending is already included in the determination of $\tilde{x}_{12}(\theta)$, so the logic is unchanged.

When the location of parties is less asymmetric, it is still the case that individuals who choose to abstain are those with the weakest net policy preferences. This group includes individuals with ideal policies in an interval around $\tilde{x}_{12}$, but also possibly individuals 
with ideal policies around $\tilde{x}_{23}$. And while it is clear that when the asymmetry is only slightly less severe abstainers must come only from around $\tilde{x}_{12}$, this turns out to be true even when the asymmetry is very small. ${ }^{13}$

Call $B(x)$ the net benefit of voting for an individual with ideal policy $x$. In the proof we show (still assuming without loss of generality that $\left|x_{3}-x_{2}\right|<\left|x_{1}-x_{2}\right|$ ) that party 2 's potential supporters' net benefit of voting is increasing in their ideological position; i.e., that $B(x)$ is increasing for all $x$ between $\tilde{x}_{12}$ and $\tilde{x}_{23}$. At the same time, party 1 's potential supporters' net benefit of voting is decreasing in their ideological position: $B(x)$ is decreasing for all $x$ between 0 and $\tilde{x}_{12}$. By symmetry of the argument, it follows that if instead $\left.\left|x_{3}-x_{2}\right|>\left|x_{1}-x_{2}\right|\right)$, then party 2's potential supporters' net benefit of voting must be decreasing in their ideological position; i.e., that $B(x)$ is decreasing for all $x$ between $\tilde{x}_{12}$ and $\tilde{x}_{23}$. It follows that if $\left|x_{3}-x_{2}\right|=\left|x_{1}-x_{2}\right|$ (as in a LS equilibrium) then all party 2 supporters must have the same net benefit of voting: $B(x)=\delta$ for all $x \in\left(\tilde{x}_{12}, \tilde{x}_{23}\right)$ and some constant $\delta$. Thus in a LS equilibrium all individuals abstaining must be supporters of party 2. How many of them abstain will be determined by an equilibration condition $(\delta=0)$, much as in an equilibrium in mixed strategies. When we move from a LS equilibrium to a slightly asymmetric configuration, this non-generic equilibration condition breaks down completely: $B(x)$ is again strictly monotonic for all supporters of party 2 , and abstention must be in some interval either around $\tilde{x}_{12}$ or $\tilde{x}_{23}$.

Having characterized abstention patterns in this setting, we now tackle the effect of abstention on campaign spending. We showed before that in the two party setting, abstention is incentive neutral for parties; i.e., the possibility of abstention does not change parties' campaign spending levels in equilibrium. The abstention patterns uncovered by Proposition 6 however, suggest that this will not be the case in asymmetric equilibria with multiple parties. In our final results, we establish this result formally. We do this in the three-party setting, in the case of a small cost of voting. We refer to this as almost-costless voting.

We define almost-costless as follows. Let $t^{*}(\tau, \mathcal{P}, \Delta)$ denote turnout in equilibrium given voting cost $\tau$, parameters $\mathcal{P}$ and party differentiation $\mathcal{D} \equiv\left\{x_{1}, \Delta_{1}, \Delta_{2}\right\}$. For any $\eta>0$, we say that voting is $\eta$-costly given $(\tau, \mathcal{P}, \mathcal{D})$ if $\tau \in T(\tau, \mathcal{P}, \mathcal{D}) \equiv\{\tau \in[0,1]: 0<$ $\left.1-t^{*}(\tau, \mathcal{P}, \mathcal{D})<\eta\right\}$. We say that voting is almost costless if it is $\eta$-costly for arbitrarily small $\eta$.

Proposition 7 Suppose that voting is voluntary and almost costless, and fix an asymmetric short-run electoral equilibria with three parties. (1) In any such equilibrium, at least one party spends more in campaigning than when voting is costless/compulsory.

\footnotetext{
${ }^{13}$ The result that all abstainers have ideal policies to the left of the median even when the location of parties is arbitrarily close to being symmetric is due to the quadratic utility assumed for voters. In the general case of a concave utility function, it is possible that both individuals to the left and right of the median abstain, but most abstainers will have ideal policies to the left of the median. This asymmetry is what is key for our results.
} 
Furthermore, (2) if

$$
\left(x_{2}-\frac{1}{2}\right)+\left(\frac{x_{3}-x_{1}}{2}\right)>\frac{1}{2},
$$

then all parties spend more resources in campaigning when voting is voluntary and almost costless than when it is costless or compulsory.

To grasp the main logic behind this result, assume without loss of generality that $\left|x_{3}-x_{2}\right|<\left|x_{1}-x_{2}\right|$, so that individuals abstaining are in a neighborhood of $\tilde{x}_{12}$. Focus on the incentives of party 1 . The total effect of an increase in campaign spending on its vote share can be divided into two components. First, as we already mentioned before, for given turnout level an increase in party 1's campaign expenditure "persuades" potential supporters of party 1 that were previously abstaining to turnout and vote, and "discourages" supporters of party 2 that were previously voting to do so. This is the local effect. Second, there is a global effect that follows from the fact that overall turnout is changing. In the case of almost costless voting we can show that the discouragement effect on party 2 supporters dominates the persuasion effect on party 1 supporters. As a result, an increase in party 1's campaign expenditures reduces turnout. Since this reduction in turnout (at party 2's expense) further increases party 1's vote share, it follows that the marginal return of campaign spending for party 1 must be higher when voting is voluntary than when it is compulsory.

Note that $\left(\frac{x_{3}-x_{1}}{2}\right)$ is a measure of polarization, and $\left(x_{2}-\frac{1}{2}\right)$ is a measure of asymmetry of parties' ideological positions. Hence, while in general the marginal return of campaign spending for party 2 and 3 can be smaller or larger, Proposition 7 states that if asymmetry and polarization are sufficiently large, then all parties spend more resources in campaigning when voting is voluntary and almost costless than when it is costless/compulsory. The results of Proposition 7 have direct consequences on the rents obtained by parties in equilibrium. We state these in the next remark.

Remark 1 Suppose voting is almost-costless and consider a short run equilibrium with three parties. If asymmetry and polarization are sufficiently large, a policy of compulsory voting increases the rents of all parties.

Notice that a policy of compulsory voting confines campaign competition to have only a local effect: by definition there is no global effect on turnout. On the other hand, we showed that if in a short-run equilibrium asymmetry and polarization are sufficiently large, the global effect of campaign spending on turnout has a pro-competitive nature. As a result, the overall effect of a policy of compulsory voting is to reduce the competition among parties. We know from our previous analysis that the higher are the rents for parties contesting the election, the higher are the incentives for new parties to enter the competition. Hence, Remark 1 identifies conditions under which, somewhat surprisingly, a policy of compulsory voting leads to qualitatively similar results on equilibrium rents (and therefore on political competition) than a campaign-finance regulation that tightens parties' spending caps: it reduces the competitiveness of the political arena and may eventually lead to an increase in the number of parties. 
While a complete characterization of equilibria with multiple parties and voluntary voting is beyond the scope of this paper, the results of this section highlight the importance of looking at total political rents - and their effect on the competitiveness of elections - when evaluating the effects of policies designed to regulate electoral competition.

\section{Conclusion}

After the last decades of advances in the theory of industrial organization, it is now evident that putting together price competition with entry of new firms is key for the analysis of regulatory and antitrust policies. Putting together campaign competition with entry of new parties is no less important for the regulation of electoral politics.

In this paper, we studied a model of elections in non-majoritarian systems that captures the link between competition in policies and competition in campaign spending. The main thrust of the paper is that it is crucial to consider the overall competitiveness of elections - resulting from both ideological differentiation and campaigning - when evaluating policies designed to regulate electoral competition.

We illustrate this point through the analysis of two major electoral institutions. First we consider campaign spending limits. We show that increasing the spending cap can reduce the set of alternatives available to voters. This result relies completely on the level of political rents up for grabs. In fact, we show that this happens even when the equilibrium number of parties is unaffected by the responsiveness of voters to political campaigning. Second, we introduce costly voting, and consider a policy of compulsory voting vis a vis voluntary voting. We show that in the special case of two parties contesting the election, abstention is incentive-neutral. With more than two parties, however, this is not generically the case. We provide conditions for which compulsory voting reduces the incentives of all parties to spend in campaigning, increasing equilibrium political rents and making the entry of new parties more attractive.

The paper opens several avenues for future research. While in this paper we confined our analysis to abstract non-majoritarian electoral systems, the analysis can be extended to other electoral institutions (see Iaryczower and Mattozzi (2009)). In addition, extending our static model to a dynamic framework would allow us to tackle how term limits and incumbency influence electoral outcomes through their effect on total political rents. The model also has important implications for applied research. Besides delivering a number of novel empirical implications, our analysis makes clear that generically, campaign spending and entry should be treated as jointly determined in equilibrium. 


\section{Appendix A: Proofs}

Proof of Proposition 1. Consider a candidate equilibrium strategy profile in which campaign constraints are not binding; i.e., $\max \left\{\theta_{1}^{*}, \theta_{2}^{*}\right\}<L$. Note that if $\tilde{x}_{12} \in(0,1)$ the vote share mapping $m_{1}\left(\theta_{1} ; \theta_{2}, x\right)$ is differentiable and the marginal vote share is given by

$$
\frac{\partial m_{1}}{\partial \theta_{1}}=\frac{\alpha v^{\prime}\left(\theta_{1}\right)}{\Delta}
$$

The fact that campaign constraints are not binding implies that the necessary first order condition must be satisfied with equality, i.e. $\alpha v^{\prime}\left(\theta_{k}^{*}\right)=\Delta C_{v}^{\prime}\left(\theta_{k}^{*}\right)$ for $k=1,2$ or

$$
\theta_{1}^{*}=\theta_{2}^{*}=\theta^{*}=\Psi^{-1}(\Delta / \alpha) .
$$

This implies that in any equilibrium with two parties in which they are not campaign constrained, there cannot be a differential in campaign investments. Moreover, it also implies that in any two-party equilibrium in which parties are not campaign constrained, they must be sufficiently ideologically differentiated, i.e. $\Delta \geq \alpha \Psi(L) .{ }^{14}$

Since $\theta_{1}^{*}=\theta_{2}^{*}$, candidates' vote shares are $m_{1}=x_{1}+\Delta / 2$, and $m_{2}=1-x_{1}-\Delta / 2$, and therefore $\Pi_{1}^{*}=x_{1}+\Delta / 2-C\left(\theta^{*}\right)$ and $\Pi_{2}^{*}=1-x_{1}-\Delta / 2-C\left(\theta^{*}\right)$ are the equilibrium rents. The equilibrium requirement that parties earn non-negative rents implies that $\left(x_{1}, \Delta\right) \in A$, where

$$
A \equiv\left\{\left(x_{1}, \Delta\right): C\left(\Psi^{-1}(\Delta / \alpha)\right)-\Delta / 2 \leq x_{1} \leq 1-\Delta / 2-C\left(\Psi^{-1}(\Delta / \alpha)\right)\right\} .
$$

Note that if there exists some pair of candidates locations $\left(x_{1}, \Delta\right) \in A$, then the symmetric configuration $x_{1}^{\prime}=1-x_{1}$ belongs to $A$ as well. It then follows from the above inequalities that the set $A$ is nonempty (i.e., there exists a pair of candidate locations $\left(x_{1}, \Delta\right)$ such that both parties earn non-negative rents in equilibrium) if and only if $C\left(\theta^{*}\right) \leq 1 / 2$. Now, suppose first that $C(L) \leq 1 / 2$. Then $C(\theta) \leq 1 / 2$ for all $\theta$, and thus the fact that $A$ is nonempty follows immediately. Thus the necessary condition for interior campaigning $\alpha \Psi(L) \leq \Delta$ is the binding constraint. As a result, when $C(L) \leq 1 / 2$ there is an equilibrium with two parties unconstrained in campaigning as long as $\alpha \Psi(L) \leq 1$. So suppose instead that $C(L)>1 / 2$. Substituting $\theta^{*}$, we can write $C\left(\theta^{*}\right) \leq 1 / 2$ as $\Delta>\alpha \Psi\left(C^{-1}(1 / 2)\right)$. Thus there is an equilibrium with two parties unconstrained in campaigning if $\alpha \Psi(L) \leq 1$ and $\alpha \Psi\left(C^{-1}(1 / 2)\right) \leq 1$. But note that $C(L)>1 / 2$ implies that $\alpha \Psi\left(C^{-1}(1 / 2)\right)>\alpha \Psi(L)$. Thus when $C(L)>1 / 2$, the requirement of non-negative rents is the binding constraint, and there is an equilibrium with two parties unconstrained in campaigning if $\alpha \Psi\left(C^{-1}(1 / 2)\right) \leq 1$.

Finally, to obtain the lower bound on differentiation, $\underline{\Delta}$, note that we have shown that when $C(L) \leq 1 / 2$, the binding constraint on differentiation is $\Delta \geq \triangleq \equiv \alpha \Psi(L)$, and

\footnotetext{
${ }^{14}$ For any given $\theta_{2}$, 1's vote share mapping $m_{1}\left(\theta_{1} ; \theta_{2}, x\right)$ has two kinks, one at $\underline{t}$ such that $m_{1}\left(\underline{t} ; \theta_{2}, x\right) \equiv 0$ and one at $\bar{t}$ such that $m_{1}\left(\bar{t} ; \theta_{2}, x\right) \equiv 1$. In fact $\underline{t}=v^{-1}\left(v\left(\theta_{2}\right)-\Delta^{2} / \bar{\alpha}\right)<\theta_{2}$ and $\bar{t}=v^{-1}\left(v\left(\theta_{2}\right)+\Delta(1-\Delta) / \alpha\right)>\theta_{2}$. Thus marginal rent is well defined, continuous and decreasing at all points $\theta_{1} \in(\underline{t}, \bar{t})$. Since the condition for non-negative rents is also imposed for equilibrium, we know that $\theta_{1}^{*}=\theta^{*}$ is indeed a best response.
} 
when $C(L)>1 / 2$, the binding constraint on differentiation is $\Delta>\triangleq \equiv \alpha \Psi\left(C^{-1}(1 / 2)\right)$. Thus $\triangleq$ is increasing in $\alpha$.

Proof of Proposition 2. Consider first $C(L) \leq 1 / 2$. Then $b=1 / \Psi(L)$ and $\alpha>b$ implies that there does not exist $\Delta \in(0,1)$ such that $\Delta \geq \alpha \Psi(L)$. It follows that in any equilibrium $\alpha \Psi(L)>\Delta$, and therefore - as long as both parties earn non-negative rents when constrained in campaigning - it must be that $\theta_{1}^{*}=\theta_{2}^{*}=L$. Recall that the condition $C(L) \leq 1 / 2$ guarantees that the set $A$ defined in the proof of Proposition 1 is nonempty. In particular, then, if $C(L) \leq 1 / 2$ and $\alpha \Psi(L)>1$ (i.e., $\alpha>b$ ) then in all equilibria the two parties are campaign-constrained. Consider now the case of $C(L)>1 / 2$. Then $b=1 / \Psi\left(C^{-1}(1 / 2)\right)$ and $\alpha>b$ implies that there does not exist $\Delta \in(0,1)$ such that $\Delta \geq \alpha \Psi\left(C^{-1}(1 / 2)\right)$. But $\Delta<\alpha \Psi\left(C^{-1}(1 / 2)\right)$ is equivalent to $C\left(\theta^{*}\right)>1 / 2$. It follows that if $C(L)>1 / 2$ and $\alpha>b$ the set $A$ is empty, and there cannot be an equilibrium in pure strategies with more than one party.

Lemma 3 In (any voting subgame of) any electoral equilibrium, voters vote for their preferred candidate.

Proof of Lemma 3. Suppose voter $i$ 's preferred party is $k^{*}(i) \in \mathcal{K}$, and that $\tilde{k} \in \mathcal{K}$ and $\tilde{k} \neq k^{*}(i)$. Let $t_{k}\left(\sigma_{-i}^{v}\right)$ denote the number of votes for party $k$ given a voting strategy profile $\sigma_{-i}^{v}$ for all voters other than $i$. The payoff for $i$ of voting for $\tilde{k}$ given $\sigma_{-i}^{v}, U\left(\tilde{k} ; \sigma_{-i}^{v}\right)$, is

$$
\sum_{k \neq \tilde{k}, k^{*}(i) \in \mathcal{K}} \frac{t_{k}\left(\sigma_{-i}^{v}\right)}{N} u\left(x_{k} ; z^{i}\right)+\frac{\left[t_{\tilde{k}}\left(\sigma_{-i}^{v}\right)+1\right]}{N} u\left(x_{\tilde{k}} ; z^{i}\right)+\frac{t_{k^{*}(i)}\left(\sigma_{-i}^{v}\right)}{N} u\left(x_{k^{*}(i)} ; z^{i}\right) .
$$

Similarly, the payoff for $i$ of voting for $k^{*}(i)$ given $\sigma_{-i}^{v}, U\left(k^{*}(i) ; \sigma_{-i}^{v}\right)$, is

$$
\sum_{k \neq \tilde{k}, k^{*}(i) \in \mathcal{K}} \frac{t_{k}\left(\sigma_{-i}^{v}\right)}{N} u\left(x_{k} ; z^{i}\right)+\frac{t_{\tilde{k}}\left(\sigma_{-i}^{v}\right)}{N} u\left(x_{\tilde{k}} ; z^{i}\right)+\frac{\left[t_{k^{*}(i)}\left(\sigma_{-i}^{v}\right)+1\right]}{N} u\left(x_{k^{*}(i)} ; z^{i}\right) .
$$

Thus

$$
U\left(k^{*}(i) ; \sigma_{-i}^{v}\right)-U\left(\tilde{k} ; \sigma_{-i}^{v}\right)=\frac{1}{N}\left[u\left(x_{k^{*}(i)} ; z^{i}\right)-u\left(x_{\tilde{k}} ; z^{i}\right)\right],
$$

which is positive by definition of $k^{*}(i)$. Since $\sigma_{-i}^{v}$ was arbitrary, this shows that voting sincerely strictly dominates voting for any other available party and is thus a dominant strategy for voter $i$. It follows that in all Nash equilibria in the voting stage voters vote sincerely among parties contesting the election.

Proof of Lemma 1. Consider first interior parties $k=2, \ldots, K-1$ and note that $m_{k}(\theta, x) \geq 0$ if and only if

$$
\theta_{k} \geq v^{-1}\left(\left(\delta_{k}^{r} v\left(\theta_{\ell(k)}\right)+\left(1-\delta_{k}^{r}\right) v\left(\theta_{r(k)}\right)\right)-\frac{\delta_{k}^{r}\left(1-\delta_{k}^{r}\right)\left(\Delta_{k}^{T}\right)^{2}}{2 \alpha}\right) \equiv \underline{\theta}_{k}\left(\theta_{-k}, x\right)
$$


But then $\underline{\theta}_{k}\left(\theta_{-k}, x\right)<\theta$ if $\theta_{\ell(k)}=\theta_{r(k)} \leq \theta$, and this is always the case in a LS equilibrium with $\theta_{k}<L$ for interior parties whose neighbors are themselves interior parties since $\theta_{\ell(k)}=\theta_{r(k)}=\theta_{k}$. Similarly, for interior parties with one extreme neighbor, say $k=K-1$, it must be that $\theta_{k}=\theta_{\ell(k)}>\theta_{r(k)}\left(\theta_{k}=\theta_{r(k)}>\theta_{\ell(k)}\right.$ for $\left.k=2\right)$. For extreme parties this is also true since in equilibrium they earn nonnegative rents, which can only happen if they choose a campaign investment above the lower discontinuity point. Now consider the upper discontinuity point and let $j>\ell>k$. We want to show that if $\theta_{r}=\theta$ for all $r \neq k$, then $k$ 's best response $\theta_{k}\left(\theta_{-k}\right)$ is lower than the point $D_{k}^{\ell, j}\left(\theta_{-k}\right)$ at which $\tilde{x}_{k \ell}=\tilde{x}_{j \ell}$. Recall that for $r>k, \tilde{x}_{k r}=\left(x_{k}+x_{r}\right) / 2+\alpha\left(v\left(\theta_{k}\right)-v\left(\theta_{r}\right)\right) /\left|x_{r}-x_{k}\right|$. After some algebra, we obtain

$$
D_{k}^{\ell, j}\left(\theta_{-k}\right)=v^{-1}\left(\frac{\left|x_{\ell}-x_{k}\right|\left|x_{j}-x_{k}\right|}{2 \alpha}-\frac{\left|x_{\ell}-x_{k}\right| v\left(\theta_{j}\right)-\left|x_{j}-x_{k}\right| v\left(\theta_{\ell}\right)}{x_{j}-x_{\ell}}\right)
$$

and if $\theta_{r}=\theta$ for all $r \neq k,(10)$ simplifies to

$$
D_{k}^{\ell, j}(\theta)=v^{-1}\left(v(\theta)+\frac{\left|x_{\ell}-x_{k}\right|\left|x_{j}-x_{k}\right|}{2 \alpha}\right)>\theta .
$$

Hence, it follows from (9) and (10) that the vote share is differentiable in $\left[\underline{\theta}_{k}(\theta, x), D_{k}(\theta)\right]$. The same logic holds for extreme parties. Suppose all interior parties $k=2, \ldots, K-1$ choose in equilibrium $\theta^{*}<L$. Consider $k^{\prime}$ s problem. Note that since $\theta_{j}^{*}=\theta_{r}^{*}$ for all $j, r \neq k$, then $k$ 's FOC is given by $2 \alpha v^{\prime}\left(\theta_{k}^{*}\right)=\Delta C_{v}^{\prime}\left(\theta_{k}^{*}\right)$. By our previous argument, this is well defined, and since the marginal vote share is decreasing above $\underline{\theta}_{k}(\theta, x)$, then the sufficient second order condition is satisfied. Therefore

$$
\theta_{k}^{*}=\theta^{*}=\Psi^{-1}\left(\frac{\Delta}{2 \alpha}\right) \text { for all } k=2, \ldots, K-1 .
$$

Finally, given that interior parties are choosing $\theta_{k}<L$, then optimal campaign spending by extreme parties must be strictly smaller that $L$ as well. In particular, it must be that $\theta_{1}^{*}=\theta_{K}^{*}=\Psi^{-1}(\Delta / \alpha)$. This completes the proof.

Proof of Proposition 3. Fix $K \geq 3$, and assume that $K \times C(L) \leq 1$. Lemma 4 shows that if $\alpha \geq \bar{a} \equiv C(L) / \Psi(L)$, there exists a short-run LS equilibrium with $K \leq$ $1 / C(L)$ parties, such that $\theta_{1}^{*}(\alpha)=\theta^{*}(\alpha)=L$. Lemma 5 shows that if $C(L) / 2 \Psi(L) \equiv$ $\underline{a} \leq \alpha \leq \bar{a}$, there exists a short-run LS equilibrium with $K \leq 1 / C(L)$ parties in which $\theta_{1}^{*}(\alpha)=\Psi^{-1}(\Delta / \alpha)<\theta^{*}(\alpha)=L$. Lemma 6 shows that if $\alpha \leq \underline{a}$, there exists a shortrun LS equilibrium with $K \leq 1 / C(L)$ parties in which $\theta_{1}^{*}(\alpha)=\Psi^{-1}(\Delta / \alpha)<\theta^{*}(\alpha)=$ $\Psi^{-1}(\Delta / 2 \alpha)<L$.

Lemma 4 Take $K \geq 3$ as given, and suppose $K \times C(L) \leq 1$. Then for any $\alpha \geq \bar{a}$, there exists a short-run LS equilibrium $\left(x_{1}^{*}(\alpha), \theta_{1}^{*}(\alpha), \Delta^{*}(\alpha), \theta^{*}(\alpha)\right)$ with $K$ parties such that $\theta^{*}(\alpha)=\theta_{1}^{*}(\alpha)=L$, and all parties earn positive rents. 
Proof of Lemma 4. Suppose that in equilibrium $\theta_{k}^{*}=L$ for $k=2, \ldots, K-1$. For $L$ to be optimal for $k$ it must be that the marginal vote share given that the other parties are also choosing $L$ is higher than the marginal cost at $L$; i.e., $2 \alpha v^{\prime}(L) / \Delta \geq C_{v}^{\prime}(L)$, or $\Delta \leq 2 \alpha \Psi(L)$. For nonnegative rents we must have $\Pi_{k}^{*}=\Delta-C(L) \geq 0$, or $\Delta \geq C(L)$. Now consider the extreme parties. For $\theta_{1}^{*}=\theta_{K}^{*}=L$, it is necessary that $\Delta \leq \alpha \Psi(L)$. For nonnegative rents it is necessary that $\Pi_{1}^{*}=x_{1}+\Delta / 2-C(L) \geq 0$, and since $\Delta \geq C(L)$ it is sufficient that $x_{1} \geq \Delta / 2$. Now $2 x_{1}+(K-1) \Delta=1$, so $x_{1}=(1-(K-1) \Delta) / 2$. Substituting, $x_{1} \geq \Delta / 2$ becomes $\Delta \leq 1 / K$. Putting everything together implies that in equilibrium $\Delta^{*} \in A_{T}$, where

$$
A_{T} \equiv\{\Delta: C(L) \leq \Delta \leq \min \{\alpha \Psi(L), 1 / K\}\}
$$

The set $A_{T}$ is nonempty if and only if $K \times C(L) \leq 1$ and $\alpha \geq C(L) / \Psi(L)=\bar{a}$. Finally, note that if $\Delta^{*}$ is in the interior of $A_{T}$ then all parties earn positive rents.

Lemma 5 Take $K \geq 3$ as given, and suppose $K \times C(L) \leq 1$. Then for any $\alpha: \underline{a} \leq$ $\alpha \leq \bar{a}$, there exists a short-run LS equilibrium $\left(x_{1}^{*}(\alpha), \theta_{1}^{*}(\alpha), \Delta^{*}(\alpha), \theta^{*}(\alpha)\right)$ with $K$ parties such that $\theta_{1}^{*}(\alpha)=\Psi^{-1}(\Delta / \alpha)<\theta^{*}(\alpha)=L$, and all parties earn positive rents.

Proof of Lemma 5. The first part of the proof is identical to the proof of Lemma 4. For $L$ to be optimal for $k=2, \ldots, K-1$ it must be that $\Delta \leq 2 \alpha \Psi(L)$, and for nonnegative rents for interior parties, we must have $\Delta \geq C(L)$. For extreme parties to choose interior campaign spending, i.e., $\theta_{1}^{*}=\theta_{K}^{*}=\Psi^{-1}(\Delta / \alpha)<L$, it must be that $\Delta>\alpha \Psi(L)$. For nonnegative rents we need

$$
\Pi_{1}^{*}=\Pi_{K}^{*}=x_{1}+\frac{\Delta}{2}-\frac{\alpha}{\Delta}\left[v(L)-v\left(\theta_{1}^{*}\right)\right]-C\left(\theta_{1}^{*}\right) \geq 0 .
$$

Since $\theta_{1}^{*}$ maximizes $\Pi_{1}\left(\theta_{1}\right)$, then $\Pi_{1}\left(\theta_{1}^{*}\right) \geq \Pi_{1}\left(\theta_{1}\right)$ for all $\theta_{1} \neq \theta_{1}^{*}$, and thus it is enough to show that $\Pi_{1}(L) \geq 0$. But this is $x_{1}+\Delta / 2 \geq C(L)$, which holds whenever $x_{1} \geq \Delta / 2$. As before, this implies $\Delta \leq 1 / K$. Putting everything together implies that in equilibrium $\Delta^{*} \in A_{M}$, where

$$
A_{M} \equiv\{\Delta: \max \{C(L), \alpha \Psi(L)\} \leq \Delta \leq \min \{2 \alpha \Psi(L), 1 / K\}\}
$$

The set $A_{M}$ is nonempty if and only if (1) $K \leq 1 / C(L),(2) \alpha \geq C(L) / 2 \Psi(L)$, and (3) $K \leq 1 / \alpha \Psi(L)$. But $\alpha \leq \bar{a}$ implies that $C(L) \geq \alpha \Psi(L)$, and the result follows. As in the previous lemma, note that if $\Delta^{*}$ is in the interior of $A_{M}$ then all parties earn positive rents.

Lemma 6 Take $K \geq 3$ as given, and suppose $K \times C(L) \leq 1$. Then

1. For any $\alpha \leq \underline{a}$, there exists a short-run LS equilibrium $\left(x_{1}^{*}(\alpha), \theta_{1}^{*}(\alpha), \Delta^{*}(\alpha), \theta^{*}(\alpha)\right)$ with $K$ parties such that $\theta_{1}^{*}(\alpha)=\Psi^{-1}(\Delta / \alpha)<\theta^{*}(\alpha)=\Psi^{-1}(\Delta / 2 \alpha)<L$, and all parties earn positive rents. 
2. For any $\alpha \leq 1 /\left(6 \Psi\left(C_{v}^{-1}(1 / 3-F)\right)\right)$, there exists a short-run $L S$ equilibrium $\left(x_{1}^{*}(\alpha), \theta_{1}^{*}(\alpha), \Delta^{*}(\alpha), \theta^{*}(\alpha)\right)$ with at least three parties in which $\theta_{k}^{*}(\alpha)<L$ for all $k$, and all interior parties earn zero rents.

Proof of Lemma 6. Consider first the interior parties $k=2, \ldots, K-1$. If $\theta_{j}^{*}=\theta_{r}^{*}<L$ for all $j, r \neq k$, then Lemma 1 implies that $k$ 's marginal vote share is differentiable, and $k$ 's FOC is given by $2 \alpha v^{\prime}\left(\theta_{k}^{*}\right) / \Delta=C_{v}^{\prime}\left(\theta_{k}^{*}\right)$. Therefore,

$$
\theta_{k}^{*}=\theta^{*}=\Psi^{-1}\left(\frac{\Delta}{2 \alpha}\right) \text { for all } k=2, \ldots, K-1
$$

Moreover, since $\theta^{*} \leq L$, it must be that $\Delta \geq 2 \alpha \Psi(L)$. Non-negative rents for interior parties require that $\Pi_{k}^{*}=\Delta-C\left(\theta^{*}\right) \geq 0$ or $\theta^{*} \leq C_{v}^{-1}(\Delta-F)$. Substituting $\theta^{*}$ we get $\Delta \geq 2 \alpha \Psi\left(C_{v}^{-1}(\Delta-F)\right)$. And note that $2 \alpha \Psi\left(C_{v}^{-1}(\Delta-F)\right) \geq 2 \alpha \Psi(L)$ if and only if $\Delta \leq$ $C(L)$. Then, as long as in equilibrium $\Delta \leq C(L)$ (i.e., $\Pi_{k}(L) \leq 0$ for $k=2, \ldots, K-1$ ), $\Delta \geq 2 \alpha \Psi\left(C_{v}^{-1}(\Delta-F)\right)$ implies $\Delta \geq 2 \alpha \Psi(L)$. That is, if interior parties earn nonnegative rents, they are choosing $\theta^{*}<L$. Therefore in equilibrium either $2 \alpha \Psi\left(C_{v}^{-1}(\Delta-F)\right) \leq$ $\Delta \leq C(L)$ or $\Delta \geq \max \{C(L), 2 \alpha \Psi(L)\}$. Consider next optimality and nonnegative rents for extreme parties. Note first that given that interior parties are choosing $\theta^{*}<L$, then optimal campaign spending by extreme parties must be interior as well. For nonnegative rents we need $\Pi_{1}^{*}=x_{1}+\Delta / 2-\alpha / \Delta\left(v\left(\theta^{*}\right)-v\left(\theta_{1}^{*}\right)\right)-C\left(\theta_{1}^{*}\right) \geq 0$. Since $\Pi_{1}^{*}$ is maximized at $\theta_{1}^{*}$, then $\Pi_{1}^{*}\left(\theta_{1}^{*}\right) \geq \Pi_{1}^{*}\left(\theta_{1}\right)$ for all $\theta_{1} \neq \theta_{1}^{*}$ and, as a result, it suffices to show that $\Pi_{1}^{*}\left(\theta^{*}\right)>0$, or equivalently, $(K-2) \Delta / 2+C\left(\theta^{*}\right) \leq 1 / 2$. But since in equilibrium it must be that $\Delta \geq C\left(\theta^{*}\right)$, then it is sufficient that $\Delta \leq 1 / K$.

Putting everything together, then in equilibrium either $2 \alpha \Psi\left(C_{v}^{-1}(\Delta-F)\right) \leq \Delta \leq$ $\min \{C(L), 1 / K\}$ or $\max \{C(L), 2 \alpha \Psi(L)\} \leq \Delta \leq 1 / K$. To conclude the proof of part (1), consider the latter case. There exists such a $\Delta$ iff (1) $K \leq 1 / C(L)$, and (2) $K \leq$ $1 /(2 \alpha \Psi(L))$. But $\alpha \leq \underline{a}$ implies $C(L) \geq 2 \alpha \Psi(L)$, and the result follows. Moreover, if the above inequalities are strict, then all parties earn positive rents. To conclude the proof of part (2), consider instead an equilibrium in which $2 \alpha \Psi\left(C_{v}^{-1}(\Delta-F)\right) \leq \Delta \leq$ $\min \{C(L), 1 / K\}$. There exists such a $\Delta$ iff $(1) K \leq 1 / C(L)$, and $(2) 2 \alpha \Psi\left(C_{v}^{-1}(\Delta-F)\right) \leq$ $\Delta$. Note that the right-hand side is increasing in $\Delta$, and the left-hand side is decreasing in $\Delta$. With $\Delta=1 / K$, this is $2 \alpha \Psi\left(C_{v}^{-1}(1 / K-F)\right) \leq 1 / K$. Note that if this is satisfied for some $K \geq 3$, it is satisfied for $K=3$. Then we need $2 \alpha \Psi\left(C_{v}^{-1}(1 / 3-F)\right) \leq 1 / 3$. But this is the same as $\alpha \leq 1 /\left(6 \Psi\left(C_{v}^{-1}(1 / 3-F)\right)\right)$.

Proof of Theorem 1. The proof follows from Propositions 8 and 9.

Proposition 8 Suppose that $2 \times C(L) \leq 1$ and $2 \times F \geq 1 / 2$. Then there exists a longrun electoral equilibrium with two parties. Moreover, there exist a threshold $\bar{\alpha}_{2 P}$ and, for any $\alpha$, a long-run two-party electoral equilibrium $\left(x_{1}^{*}(\alpha), \Delta^{*}(\alpha), \theta^{*}(\alpha)\right)$ such that 
1. If $\alpha \leq \bar{\alpha}_{2 P}$, then both parties campaign unconstrained, and campaign spending is decreasing in parties' ideological differentiation and voters' ideological focus i.e., $\theta^{*}(\alpha)=\Psi^{-1}(\Delta / \alpha)<L$.

2. If $\alpha>\bar{\alpha}_{2 P}$, then both parties are campaign constrained, i.e., $\theta^{*}(\alpha)=L$.

Proof of Proposition 8. Follows from Lemma 2 (in the text) and Lemma 7.

Lemma 7 Suppose that $2 \times C(L) \leq 1$ and $2 \times F \geq 1 / 2$. Then for any $\alpha>\bar{\alpha}_{2 P}$ there exists a long-run two-party electoral equilibrium $\left(x_{1}^{*}(\alpha), \Delta^{*}(\alpha), \theta^{*}(\alpha)\right)$ such that both parties are campaign-constrained; i.e., $\theta^{*}(\alpha)=L$.

Proof of Lemma 7. We showed in the proof of Proposition 2 that if two parties compete for votes in the election, and (i) voters vote for their preferred party, (ii) both parties are constrained in campaign spending; i.e., $\theta^{*}=L$, and (iii) parties' ideological differentiation is $\Delta<\alpha \Psi(L)$, then there is a location of the left party $x_{1}$ such that a short-run electoral equilibrium exists. We next show that if conditions (i) and (ii) are satisfied, and parties' ideological differentiation $\Delta \in B_{2 P}^{*}$, where

$$
B_{2 P}^{*} \equiv\left\{\Delta: 1-2 F \leq \Delta \leq \min \left\{\alpha \Psi(L), 2 C(L), 1-2 C_{v}(L)\right\}\right\},
$$

then there is a location of the left party $x_{1}$ such that a long-run electoral equilibrium exists. It is then easy to check that the conditions in the hypothesis imply that $B_{2 P}^{*}$ is nonempty. Consider then the threat of entry. Suppose $j$ enters at $x_{j} \in\left(0, x_{1}\right)$. As before, $\hat{\theta}_{1}=\hat{\theta}_{2}=L$ is a mutual best response if $\Delta / 2 \geq C_{v}(L)$. Now, given that $\hat{\theta}_{1}=\hat{\theta}_{2}=\hat{\theta}_{j}=L$ we have that $\tilde{r}_{j}=\left(x_{j}+x_{2}\right) / 2$ and $\tilde{\ell}_{j}=\left(x_{1}+x_{j}\right) / 2$. But then

$$
\Pi_{j}\left(\hat{\theta}_{j}\right)=\frac{\Delta}{2}-C(L)<0 \Leftrightarrow \Delta<2 C(L)
$$

Since $\hat{\theta}_{1}=\hat{\theta}_{2}=L$, a sufficient condition to deter entry in $\left[0, x_{1}\right]$ is $x_{1} \leq F$. Similarly $1-x_{2} \leq F$ prevents entry in $\left[1-x_{2}, 1\right]$. Since $\Delta=1-\left[x_{1}+\left(1-x_{2}\right)\right]$, this requires $\Delta \geq 1-2 F$. This gives $B_{2 P}^{*}$.

Proposition 9 Take $K \geq 3$ as given. If $K \times C(L) \leq 1$, and $K \times F \geq 1 / 2$ there exists a long-run $L S$ equilibrium with $K$ parties. Moreover, there exist $\underline{\alpha}, \bar{\alpha}$ and, for any $\alpha$ a long-run LS equilibrium $\left(x_{1}^{*}(\alpha), \theta_{1}^{*}(\alpha), \Delta^{*}(\alpha), \theta^{*}(\alpha)\right)$ such that

1. If $\alpha \leq \underline{\alpha}$, then all parties campaign unconstrained, and for every party $k$, campaign spending is decreasing in parties' ideological differentiation and voters' ideological focus i.e., $\theta_{1}^{*}(\alpha)=\Psi^{-1}(\Delta / \alpha)<\theta^{*}(\alpha)=\Psi^{-1}(\Delta / 2 \alpha)<L$.

2. If $\underline{\alpha} \leq \alpha \leq \bar{\alpha}$, then only interior parties are campaign constrained, i.e., $\theta_{1}^{*}(\alpha)=$ $\Psi^{-1}(\Delta / \alpha)<\theta^{*}(\alpha)=L$, and if $\alpha \geq \bar{\alpha}$ all parties are campaign constrained, i.e., $\theta_{1}^{*}(\alpha)=\theta^{*}(\alpha)=L$. 
Proof of Proposition 9. Fix $K \geq 3$, and assume that $K \times C(L) \leq 1$ and $K \times F \leq 1 / 2$. Lemma 8 extends Lemma 4 to long-run equilibria, and shows that if

$$
\alpha \geq \max \left\{\frac{C(L)}{\Psi(L)}, \frac{1-2 F}{(K-1) \Psi(L)}\right\} \equiv \bar{\alpha},
$$

there exists a long-run LS equilibrium with $K$ parties, such that $\theta_{1}^{*}(\alpha)=\theta^{*}(\alpha)=L$. Similarly, Lemma 9 extends Lemma 5 to long-run equilibria, and shows that if

$$
\underline{\alpha} \equiv \max \left\{\frac{1-2 F}{2 \Psi(L)(K-1)}, \frac{C(L)}{2 \Psi(L)}\right\} \leq \alpha \leq \bar{\alpha},
$$

there exists a long-run LS equilibrium with $K$ parties in which $\theta_{1}^{*}(\alpha)=\Psi^{-1}(\Delta / \alpha)<$ $\theta^{*}(\alpha)=L$. Finally, Lemma 10 extends the corresponding Lemma 6 to long-run equilibria and shows that if $\alpha \leq \underline{\alpha}$, there exists a long-run LS equilibrium with $K$ parties in which $\theta_{1}^{*}(\alpha)=\Psi^{-1}(\Delta / \alpha)<\theta^{*}(\alpha)=\Psi^{-1}(\Delta / 2 \alpha)<L$.

Lemma 8 Take $K \geq 3$ as given. If $K \times C(L) \leq 1$ and $K \times F \geq 1 / 2$, then for any $\alpha \geq \bar{\alpha}$, there exists a LS equilibrium $\left(x_{1}^{*}(\alpha), \theta_{1}^{*}(\alpha), \Delta^{*}(\alpha), \theta^{*}(\alpha)\right)$ with $K$ parties such that $\theta^{*}(\alpha)=\theta_{1}^{*}(\alpha)=L$, and all parties earn positive rents.

Proof of Lemma 8. We showed in the proof of Lemma 4 that if (i) voters vote for their preferred party, (ii) all parties are campaign constrained, i.e., $\theta_{k}^{*}=L$ for all $k$, and (iii) parties' ideological differentiation $\Delta \in A_{T}=\{\Delta: C(L) \leq \Delta \leq \min \{\alpha \Psi(L), 1 / K\}\}$, then a short-run LS electoral equilibrium exists. We show below that if conditions (i) and (ii) are satisfied, and (iii') parties' ideological differentiation $\Delta \in A_{T}^{*} \subset A_{T}$, where

$$
A_{T}^{*} \equiv\left\{\Delta: \max \left\{\frac{1-2 F}{(K-1)}, C(L)\right\} \leq \Delta \leq \min \left\{2 C(L), \alpha \Psi(L), \frac{1}{K}\right\}\right\},
$$

then a long-run electoral equilibrium exists. Moreover, if the inequalities are strict (if $\Delta$ is in the interior of $A_{T}^{*}$ ) then all parties earn positive rents. Note that $A_{T}^{*}$ is nonempty iff

$$
\alpha \geq \max \left\{\frac{C(L)}{\Psi(L)}, \frac{1-2 F}{K-1 \Psi(L)}\right\}=\bar{\alpha}
$$

and

$$
\max \left\{\frac{C_{v}(L)+1 / 2}{C(L)}, \frac{1}{2 F}\right\} \leq K \leq \frac{1}{C(L)} .
$$

Now, $\left(C_{v}(L)+1 / 2\right) / C(L) \leq 1 / 2 F$ if and only if $F \leq 1 / 2$, but this must surely be the case, since for $K \geq 3$, the RHS of (13) implies $C(L) \leq 1 / 3$. Thus (13) boils down to $K C(L) \leq 1$ and $K F \geq 1 / 2$. Consider then the entry of $j$ at $x_{j} \in\left(x_{k}, x_{k+1}\right)$ for $k=1, \ldots, K-1$. Since in equilibrium $\theta_{k}^{*}=L$ for $k=1, \ldots, K$, then as long as incumbents do not prefer to quit campaigning after $j$ 's entry, we can always sustain in the continuation game an equilibrium such that $\hat{\theta}_{j}=\hat{\theta}_{k}=L$ for all $j, k$. For incumbents to prefer not to quit campaigning it is enough that $\Delta / 2 \geq C_{v}(L)$, or $\Delta \geq 2 C_{v}(L)$. But since $C_{v}(L)<F$ by 
hypothesis, this is implied by $\Delta>C(L)$. Given $\hat{\theta}_{j}=\hat{\theta}_{k}=L, j$ 's entry is not profitable if $\hat{\Pi}_{j}=\Delta / 2-C(L)<0$, or equivalently $\Delta<2 C(L)$. For no profitable entry of (more) extreme parties it is enough that $x_{1} \leq F$, since $\hat{\theta}_{j} \leq \hat{\theta}_{1}$ implies that in the continuation after entry $\tilde{x}_{1 j}<\left(x_{1}+x_{j}\right) / 2<x_{1}$. Substituting $x_{1}=1-x_{K}=(1-(K-1) \Delta) / 2$, this is $\Delta \geq(1-2 F) /(K-1)$. Together, these conditions imply that in equilibrium it must be that $\Delta \in A_{T}^{*}$.

Lemma 9 Take $K \geq 3$ as given. If $K \times C(L) \leq 1$ and $K \times F \geq 1 / 2$, then for any $\alpha \in[\underline{\alpha}, \bar{\alpha}]$, there exists a LS equilibrium $\left(x_{1}^{*}(\alpha), \theta_{1}^{*}(\alpha), \Delta^{*}(\alpha), \theta^{*}(\alpha)\right)$ with $K$ parties such that $\theta_{1}^{*}(\alpha)=\Psi^{-1}(\Delta / \alpha)<\theta^{*}(\alpha)=L$, and all parties earn positive rents.

Proof. We showed in Lemma 5 that if (i) voters vote for their preferred party, (ii) parties are campaign constrained if and only if they are not extreme, i.e., $\theta_{k}^{*}=L$ if and only if $k=2, \ldots, K-1$, and (iii) parties' ideological differentiation $\Delta \in A_{M}=$ $\{\Delta: \max \{C(L), \alpha \Psi(L)\} \leq \Delta \leq \min \{2 \alpha \Psi(L), 1 / K\}\}$, then a a short-run LS electoral equilibrium exists. We next show that if conditions (i) and (ii) are satisfied, and parties' ideological differentiation $\Delta \in A_{M}^{*} \subset A_{M}$, where

$A_{M}^{*} \equiv\left\{\Delta: \max \left\{\frac{1-2 F}{K-1}, C(L), \alpha \Psi(L)\right\} \leq \Delta \leq \min \left\{2 C(L), \frac{1-2 C_{v}(L)}{K-1}, \frac{1}{K}, 2 \alpha \Psi(L)\right\}\right\}$,

then a long-run electoral equilibrium exists. Moreover, if the inequalities are strict (if $\Delta$ is in the interior of $A_{M}^{*}$ ) then all parties earn positive rents. Note that since $C(L) \leq 1 / K$ and $F \geq 1 / 2 K$ imply that $\left(1-2 C_{v}(L)\right) /(K-1) \geq 1 / K$, the conditions defining $A_{M}^{*}$ are equivalent to those defining $A_{T}^{*}$ in all but the $\alpha$ terms. It follows from this that $A_{M}^{*}$ is nonempty if and only if

$$
\frac{1}{2 F} \leq K \leq \frac{1}{C(L)}
$$

and

$$
\max \left\{\frac{(1-2 F)}{2 \Psi(L)(K-1)}, \frac{C(L)}{2 \Psi(L)}\right\} \leq \alpha \leq \min \left\{\frac{1}{K \Psi(L)}, \frac{1-2 C_{v}(L)}{(K-1) \Psi(L)}, \frac{2 C(L)}{\Psi(L)}\right\} .
$$

Now, the LHS of (14) is equal to $\underline{\alpha}$ by definition. Simple algebra shows (using the conditions $C(L) K \leq 1$ and $K F \geq 1 / 2)$ that the RHS of (14) is bigger than $\bar{\alpha}$. Consider then the threat of entry. As in the previous lemma, to deter entry by $j$ at $x_{j} \in\left(x_{k}, x_{k+1}\right)$ it is enough that $2 C_{v}(L) \leq \Delta<2 C(L)$ and $C_{v}(L) \leq x_{1}$. To deter entry of more extreme parties, a sufficient condition is $x_{1} \leq F$, and $2 C_{v}(L) \leq \Delta$ (recall that this guarantees that the extreme incumbent parties will not quit campaigning in the continuation game). Since $C_{v}(L) \leq F$ by hypothesis, this is implied by $\Delta \geq C(L)$. Substituting $\Delta_{0}=$ $(1-(K-1) \Delta) / 2$ and collecting the relevant inequalities gives $A_{M}^{*}$.

Lemma 10 Take $K \geq 3$ as given. If $K \times C(L) \leq 1$ and $K \times F \geq 1 / 2$, then for any $\alpha \leq \underline{\alpha}$ there exists a LS equilibrium $\left(x_{1}^{*}(\alpha), \theta_{1}^{*}(\alpha), \Delta^{*}(\alpha), \theta^{*}(\alpha)\right)$ with $K$ parties such that $\theta_{1}^{*}(\alpha)=\Psi^{-1}(\Delta / \alpha)<\theta^{*}(\alpha)=\Psi^{-1}(\Delta / 2 \alpha)<L$, and all parties earn positive rents. 
Proof. We showed in Lemma 6 that if (i) voters vote for their preferred candidate, (ii) parties are unconstrained in campaign spending and choose

$$
\theta_{k}^{*}=\theta^{*}=\Psi^{-1}\left(\frac{\Delta}{2 \alpha}\right) \text { for all } k=2, \ldots, K-1, \text { and } \theta_{1}^{*}=\theta_{K}^{*}=\Psi^{-1}\left(\frac{\Delta}{\alpha}\right),
$$

and (iii) candidate's differentiation $\Delta \in A_{L} \equiv\{\Delta: \max \{C(L), 2 \alpha \Psi(L)\} \leq \Delta \leq 1 / K\}$, the polity is in a short-run LS electoral equilibrium. We next show that if conditions (i) and (ii) are satisfied, and (iii') candidate's differentiation $\Delta \in A_{L}^{*} \subset A_{L}$, where

$$
A_{L}^{*} \equiv\left\{\left\{2 C_{v}(L), \frac{1-2 F}{K-1}, C(L), 2 \alpha \Psi(L)\right\} \leq \Delta \leq\left\{2 C(L), \frac{1-2 C_{v}(L)}{K-1}, \frac{1}{K}, 2 F\right\}\right\}
$$

then a long-run electoral equilibrium. Moreover, if the inequalities are strict (if $\Delta$ is in the interior of $A_{M}^{*}$ ) then all parties earn positive rents. Note that the conditions defining $A_{L}^{*}$ are equivalent to those defining $A_{M}^{*}$ in all but the $\alpha$ terms and the $2 F$ term on the RHS (recall that $C(L) \leq 1 / K$ and $F \geq 1 / 2 K$ imply $\left.2 C_{v}(L)<C_{(} L\right)$ ). Thus it follows that $A_{L}^{*}$ is nonempty if

$$
\frac{1}{2 F} \leq K \leq \frac{1}{C_{v}(L)+F}
$$

and

$$
\alpha \leq \min \left\{\frac{C(L)}{\Psi(L)}, \frac{1-2 C_{v}(L)}{2(K-1) \Psi(L)}, \frac{1}{2 K \Psi(L)}, \frac{F}{\Psi(L)}\right\} .
$$

Simple algebra shows (using the conditions $C(L) K \leq 1$ and $K F \geq 1 / 2$ ) that the RHS of (15) is bigger than $\underline{\alpha}$. Consider then the threat of entry. To deter entry of more extreme parties it is sufficient that $x_{1}<F$, and since $x_{1}=(1-(K-1) \Delta) / 2$ this can be written as $\Delta>(1-2 F) /(K-1)$. So suppose that $j$ enters at $x_{j} \in\left(x_{k}, x_{k+1}\right)$ for $k=1, \ldots, K-1$, and define $\delta_{j}^{r} \equiv\left(x_{k+1}-x_{j}\right) / \Delta$. Suppose first that in the continuation $\hat{\theta}_{k}=\hat{\theta}_{k+1}=\hat{\theta}_{j}=L$. Then it must be that

$$
\alpha v^{\prime}(L)\left[\frac{1}{\delta_{j}^{r} \Delta}+\frac{1}{\Delta}\right] \geq C_{v}^{\prime}(L) \text { and } \alpha v^{\prime}(L)\left[\frac{1}{\left(1-\delta_{j}^{r}\right) \Delta}+\frac{1}{\Delta}\right] \geq C_{v}^{\prime}(L) .
$$

Then if $\delta_{j}^{r} \geq 1 / 2\left(j\right.$ enters in $\left(x_{k}, x_{k+1}\right)$ closer to $x_{k}$ than to $\left.x_{k+1}\right)$ the first two inequalities above hold if and only if $\Delta \leq \alpha \Psi(L)\left(1+1 / \delta_{j}^{r}\right)$, or $\delta_{j}^{r} \leq \alpha \Psi(L) /(\Delta-\alpha \Psi(L))$. Thus, the continuation strategy profile is a Nash equilibrium for $1 / 2 \leq \delta_{j}^{r} \leq \alpha \Psi(L) /(\Delta-\alpha \Psi(L))$, which is feasible if and only if $\Delta \leq 3 \alpha \Psi(L)$. When instead $\delta_{j}^{r} \leq 1 / 2(j$ enters closer to $\left.x_{k}\right)$ then we need $\Delta \leq \alpha \Psi(L)\left(1+1 /\left(1-\delta_{j}^{r}\right)\right)$, or $\delta_{j}^{r} \geq(\Delta-2 \alpha \Psi(L)) /(\Delta-\alpha \Psi(L))$. Thus, the continuation strategy profile is a Nash equilibrium for $(\Delta-2 \alpha \Psi(L)) /(\Delta-\alpha \Psi(L)) \leq$ $\delta_{j}^{r} \leq 1 / 2$, which is feasible if and only if $\Delta \leq 3 \alpha \Psi(L)$. Therefore, the strategy profile $\hat{\theta}_{k}=\hat{\theta}_{k+1}=\hat{\theta}_{j}=L$ is a Nash equilibrium in the continuation for entrants such that

$$
\frac{\Delta-2 \alpha \Psi(L)}{\Delta-\alpha \Psi(L)} \leq \delta_{j}^{r} \leq \frac{\alpha \Psi(L)}{\Delta-\alpha \Psi(L)},
$$

where $2 \alpha \Psi(L)<\Delta \leq 3 \alpha \Psi(L)$. Since the entrant in this case obtains $\Pi_{j}\left(\hat{\theta}_{j}\right)=\Delta / 2-$ $C(L)$, then as long as in equilibrium $\Delta<2 C(L)$, entry in an "interior" region as in 
(16) is not profitable. It should be clear that this rules out "interior" entrants only, since $2 \alpha \Psi(L)<\Delta$ with (16) implies that $\delta_{j}^{r} \in(0,1)$. Consider then $\delta_{j}^{r}>(\alpha \Psi(L)) /(\Delta-\alpha \Psi(L))$ ( $j$ enters close to $x_{k}$; the other case is symmetric). Consider the continuation $\hat{\theta}_{k}=\hat{\theta}_{j}=L$, $\hat{\theta}_{k+1}=\Psi^{-1}\left(\delta_{j}^{r} \Delta /\left(\left(1+\delta_{j}^{r}\right) \alpha\right)\right)<L$. This is clearly an equilibrium in the continuation $(j$ and $k$ have even a greater incentive to choose $L$ than in the previous case since they are now closer substitutes). For entry not to be profitable, we need

$$
\Pi_{j}\left(\hat{\theta}_{j}\right)=\frac{\Delta}{2}+\frac{\alpha}{\delta_{j}^{r} \Delta}\left[v(L)-v\left(\hat{\theta}_{k+1}\right)\right]-C(L)<0 .
$$

A sufficient condition for the above inequality to be true is $\Delta \leq 2 F$. To see this, suppose that the division of the electorate between $k$ and $j$ were fixed, with cutpoint $\tilde{x}_{k j}=\left(x_{k}+x_{j}\right) / 2$. Then $j$ would optimally choose $\tilde{\theta}_{j}=\Psi^{-1}\left(\delta_{j}^{r} \Delta / \alpha\right)<\hat{\theta}_{k+1}$, and we have that

$$
\Pi_{j}\left(\hat{\theta}_{j}\right) \leq \frac{\Delta}{2}-\frac{\alpha}{\delta_{j}^{r} \Delta}\left[v\left(\hat{\theta}_{k+1}\right)-v\left(\tilde{\theta}_{j}\right)\right]-\left[C_{v}\left(\tilde{\theta}_{j}\right)+F\right]<\frac{\Delta}{2}-\left[C_{v}\left(\tilde{\theta}_{j}\right)+F\right] .
$$

To assure that all incumbent parties do not prefer to quit campaigning upon entry in any continuation, it is sufficient that $\min \left\{x_{1}, \Delta / 2\right\} \geq C_{v}(L)$. Since $2 x_{1}+(K-1) \Delta=1$, then $x_{1}=(1-(K-1) \Delta) / 2$, and the previous condition can be written as

$$
2 C_{v}(L) \leq \Delta \leq \frac{1-2 C_{v}(L)}{K-1}
$$

Collecting the relevant inequalities gives $A_{L}^{*}$.

Proof of Proposition 4. In the proof of Lemma 2 we showed that $\Delta<1-2 C_{v}(L)$ is a sufficient condition to guarantee that incumbent parties do not quit campaigning upon entry of a centrist challenger. It turns out that, in all electoral equilibria where incumbent parties earn positive rents, this condition is also necessary for existence of a long-run purestrategy electoral equilibrium. To see why this is the case, first notice that the definition of long-run equilibrium requires incumbent parties to deter the entry of any challenger, and therefore also the entry of a centrist challenger championing an ideological position $x_{j}$ arbitrarily close to an incumbent's position. For example, suppose that $x_{j}>x_{1}$ but $x_{j} \rightarrow x_{1}$, and therefore the entrant and party 1 are almost "perfect substitutes" in the voters' eyes. In this case it cannot be that both $j$ and 1 choose an interior campaign spending in the continuation game. In fact, if that were the case either party could deviate and increase discretely its vote share by increasing its campaign spending slightly above the opponent's level. As a consequence, in any continuation equilibrium in pure strategies upon entry of a close centrist challenger, either both parties are campaign constrained at $L$ or the incumbent keeps spending at his equilibrium level $\theta^{*}$ and the entrant optimally chooses not to campaign. The latter case, however, cannot be optimal either when the entrant is arbitrarily close to an incumbent party earning positive rents in equilibrium. In fact the entrant can always choose a campaign level slighty above its closest competitor and de facto attract the votes of all the incumbent supporters. Since the incumbent was 
earning strictly positive rents in equilibrium, this strategy is indeed profitable for the entrant. Summarizing, when incumbent parties are earning positive rents in equilibrium, it must be the case that upon entry of a centrist challenger very close to an incumbent party, the only continuation equilibrium in pure strategies has both parties constrained in campaigning, which implies that $\Delta<1-2 C_{v}(L)$ becomes a necessary condition to guarantee that incumbent parties do not quit campaigning in the continuation game.

Since the necessary condition $\Delta<1-2 C_{v}(L)$ provides un upper bound on differentiation as a function of the level of campaign regulation, it is natural to ask whether there exist also a lower bound on ideological differentiation between parties. To answer this question, notice that when $\Delta<\alpha \Psi(L)$ it must be the case that parties are costrained in campaigning in equilibrium. This implies that when incumbents are relatively close ideologically we can use an argument similar to the one above and conclude that, upon entry of an extreme challenger $\left(x_{j}<x_{1}\right.$ or $\left.x_{2}>x_{j}\right)$ that is arbitrarily close to an incumbent, $\Delta>2 C_{v}(L)$ guarantees that incumbents do not quit campaigning in any continuation game. When voters are sufficiently responsive to campaign, i.e., when $\alpha>2 C_{v}(L) / \Psi(L)$, we have that $2 C_{v}(L)<\alpha \Psi(L)$ and hence $\Delta>2 C_{v}(L)$ becomes a necessary condition when parties are relatively close ideologically, i.e., when $\Delta<\alpha \Psi(L)$. Summarizing, when incumbent parties are earning positive rents in equilibrium and voters' are sufficiently responsive to campaigning it must be that $2 C_{v}(L)<\Delta<1-2 C_{v}(L)$ and, as campaign limits become less and less stringent, it is immediate to verify that parties' ideological positions must converge to $x_{1}=1-x_{2}=1 / 4$.

Proof of Proposition 5. We already proved in the text the second part of the proposition. To prove the first part, consider two parties representing policy positions $x_{1}, x_{2}=x_{1}+\Delta$ with campaign investment $\theta_{1}$ and $\theta_{2}$, and such that $n_{1} \leq N \tilde{x}_{12}$ voters vote for party $1, n_{2} \leq N\left(1-\tilde{x}_{12}\right)$ vote for party 2 , respectively. We show that abstainers must be be centrist. We begin by computing the net benefit of voting for a voter with ideal policy $x \leq \tilde{x}_{12}$ in a two-party equilibrium. Given a total turnout of $n_{1}+n_{2} \leq N$ voters, the expected utility of $x<\tilde{x}_{12}$ is

$$
\frac{n_{1} u\left(\theta_{1}, x_{1} ; x\right)+n_{2} u\left(\theta_{2}, x_{2} ; x\right)}{n_{1}+n_{2}}-\tau,
$$

if he votes (for party 1), and

$$
\frac{\left(n_{1}-1\right) u\left(\theta_{1}, x_{1} ; x\right)+n_{2} u\left(\theta_{2}, x_{2} ; x\right)}{n_{1}+n_{2}-1}
$$

if he does not vote. Hence, the net benefit of voting for $x<\tilde{x}_{12}$ is

$$
\frac{n_{2}\left(u\left(\theta_{1}, x_{1} ; x\right)-u\left(\theta_{2}, x_{2} ; x\right)\right)}{\left(n_{1}+n_{2}\right)\left(n_{1}+n_{2}-1\right)}-\tau .
$$

Since $u\left(\theta_{i}, x_{i} ; x\right)-u\left(\theta_{j}, x_{j} ; x\right)=2\left(x_{j}-x_{i}\right)\left(\tilde{x}_{i j}-x\right)$, the last expression simplifies to

$$
2 \frac{n_{2} \Delta\left(\tilde{x}_{12}-x\right)}{\left(n_{1}+n_{2}\right)\left(n_{1}+n_{2}-1\right)}-\tau
$$


which is decreasing in $x<\tilde{x}_{12}$. Likewise, we can compute the net benefit of voting for $x>\tilde{x}_{12}$ (a potential supporter of party 2 ), which equals

$$
2 \frac{n_{1} \Delta\left(x-\tilde{x}_{12}\right)}{\left(n_{1}+n_{2}\right)\left(n_{1}+n_{2}-1\right)}-\tau
$$

and it is increasing in $x>\tilde{x}_{12}$. As a consequence, abstainers must have ideological positions in an interval $\left(\tilde{x}_{12}^{l}, \tilde{x}_{12}^{r}\right)$ around that of the individual who is indifferent between parties, i.e. $\tilde{x}_{12}$. Hence, abstainers must be centrists. From (17) and (18), we obtain that $\tilde{x}_{12}^{l}$, and $\tilde{x}_{12}^{r}$ are given by the system of equations

$$
\left\{\begin{array}{c}
2 \frac{\left(1-\tilde{x}_{12}^{r}\right) \Delta\left(\tilde{x}_{12}-\tilde{x}_{12}^{l}\right)}{N\left(\tilde{x}_{12}^{l}+1-\tilde{x}_{12}^{r}\right)\left(\tilde{x}_{12}^{l}+1-\tilde{x}_{12}^{r}-\frac{1}{N}\right)}=\tau \\
2 \frac{\tilde{x}_{12}^{l} \Delta\left(\tilde{x}_{12}^{r}-\tilde{x}_{12}\right)}{N\left(\tilde{x}_{12}^{l}+1-\tilde{x}_{12}^{r}\right)\left(\tilde{x}_{12}^{l}+1-\tilde{x}_{12}^{r}-\frac{1}{N}\right)}=\tau
\end{array}\right.
$$

which is identical to system (7). Since we did not assume symmetry, it follows that location symmetry does not play any role and all results derived in the text hold for all short-run electoral equilibria with two parties.

Proof of Proposition 6. Consider an electoral equilibrium with three parties representing policy positions $x_{1}=\Delta_{0}, x_{2}=x_{1}+\Delta_{1}$ and $x_{3}=x_{2}+\Delta_{2}$, and campaign investment $\theta_{1}, \theta_{2}$ and $\theta_{3}$. For $j=1,2,3$, let $n_{j}$ denote the number of voters voting for party $j$. Consider first the problem of a voter with ideal policy $x \leq \tilde{x}_{12}$. The expected payoff of voting (for party 1 ) is

$$
\frac{\sum_{i=1}^{3} n_{i} u\left(\theta_{i}, x_{i} ; x\right)}{\sum_{i=1}^{3} n_{i}}-\tau
$$

On the other hand, the expected payoff of abstaining is

$$
\frac{\left(n_{1}-1\right) u\left(\theta_{1}, x_{1} ; x\right)+\sum_{i=2}^{3} n_{i} u\left(\theta_{i}, x_{i} ; x\right)}{\sum_{i=1}^{3} n_{i}-1} .
$$

Hence the net benefit of voting is

$$
\frac{\left(n_{2}+n_{3}\right)\left(u\left(\theta_{1}, x_{1} ; x\right)-u\left(\theta_{2}, x_{2} ; x\right)\right)+n_{3}\left(u\left(\theta_{2}, x_{2} ; x\right)-u\left(\theta_{3}, x_{3} ; x\right)\right)}{\left(\sum_{i=1}^{3} n_{i}\right)\left(\sum_{i=1}^{3} n_{i}-1\right)}-\tau .
$$

Since $u\left(\theta_{i}, x_{i} ; x\right)-u\left(\theta_{j}, x_{j} ; x\right)=2\left(x_{j}-x_{i}\right)\left(\tilde{x}_{i j}-x\right)$, the last expression simplifies to

$$
A(x) \equiv 2 \frac{\left(n_{2}+n_{3}\right) \Delta_{1}\left(\tilde{x}_{12}-x\right)+n_{3} \Delta_{2}\left(\tilde{x}_{23}-x\right)}{\left(\sum_{i=1}^{3} n_{i}\right)\left(\sum_{i=1}^{3} n_{i}-1\right)}-\tau .
$$

Likewise, we can compute the net benefit of voting for an individual with ideal policy $x \in\left(\tilde{x}_{12}, \tilde{x}_{23}\right)$ (a potential supporter of party 2 ). This is given by

$$
B(x) \equiv 2 \frac{n_{1} \Delta_{1}\left(x-\tilde{x}_{12}\right)+n_{3} \Delta_{2}\left(\tilde{x}_{23}-x\right)}{\left(\sum_{i=1}^{3} n_{i}\right)\left(\sum_{i=1}^{3} n_{i}-1\right)}-\tau,
$$


Finally, the net benefit of voting for an individual with ideal policy $x \in\left(\tilde{x}_{23}, 1\right)$ (a potential supporter of party 3 ) is

$$
C(x) \equiv 2 \frac{\left(n_{1}+n_{2}\right) \Delta_{2}\left(x-\tilde{x}_{23}\right)+n_{1} \Delta_{1}\left(x-\tilde{x}_{12}\right)}{\left(\sum_{i=1}^{3} n_{i}\right)\left(\sum_{i=1}^{3} n_{i}-1\right)}-\tau .
$$

Note that $A(x)$ is decreasing in $x$ for all $x \leq \tilde{x}_{12}, C(x)$ is increasing in $x$ for all $x \in\left(\tilde{x}_{23}, 1\right)$, and $B(x)$ is increasing in $x$ for all $x \in\left(\tilde{x}_{12}, \tilde{x}_{23}\right)$ if $n_{1} \Delta_{1}>n_{3} \Delta_{2}$, and decreasing in $x$ for all $x \in\left(\tilde{x}_{12}, \tilde{x}_{23}\right)$ otherwise. We then have three cases:

i) $n_{1} \Delta_{1}>n_{3} \Delta_{2}$. In this case $\min \left\{C(x): x \in\left(\tilde{x}_{23}, 1\right)\right\}=C\left(\tilde{x}_{23}\right) \geq \max \{B(x): x \in$ $\left.\left(\tilde{x}_{12}, \tilde{x}_{23}\right)\right\}=B\left(\tilde{x}_{23}\right) \geq \min \left\{B(x): x \in\left(\tilde{x}_{12}, \tilde{x}_{23}\right)\right\}=B\left(\tilde{x}_{12}\right)=\min \{A(x): x \leq$ $\left.\tilde{x}_{12}\right\}=A\left(\tilde{x}_{12}\right)$. As a consequence there will be abstention in a neighborhood of $\tilde{x}_{12}$, and $n_{1} \Delta_{1}>n_{3} \Delta_{2}$ can be rewritten as $\tilde{x}_{12}^{\ell} \Delta_{1}>\left(1-\tilde{x}_{23}\right) \Delta_{2}$.

ii) $n_{1} \Delta_{1}<n_{3} \Delta_{2}$. In this case $\min \left\{A(x): x \leq \tilde{x}_{12}\right\}=A\left(\tilde{x}_{12}\right) \geq \max \{B(x): x \in$ $\left.\left(\tilde{x}_{12}, \tilde{x}_{23}\right)\right\}=B\left(\tilde{x}_{12}\right) \geq \min \left\{B(x): x \in\left(\tilde{x}_{12}, \tilde{x}_{23}\right)\right\}=B\left(\tilde{x}_{23}\right)=\min \{C(x): x \in$ $\left.\left(\tilde{x}_{23}, 1\right)\right\}=C\left(\tilde{x}_{23}\right)$. As a consequence there will be abstention in a neighborhood of $\tilde{x}_{23}$, and $n_{1} \Delta_{1}<n_{3} \Delta_{2}$ can be rewritten as $\tilde{x}_{12} \Delta_{1}<\left(1-\tilde{x}_{23}^{r}\right) \Delta_{2}$.

iii) $n_{1} \Delta_{1}=n_{3} \Delta_{2}$. In this case $B(x)=B$ for all $x \in\left(\tilde{x}_{12}, \tilde{x}_{23}\right)$, and $B<\min \{A(x): x \leq$ $\left.\tilde{x}_{12}\right\}, B<\min \left\{C(x): x \in\left(\tilde{x}_{23}, 1\right)\right\}$. This implies that there cannot be abstainers among the supporters of the extreme parties otherwise all centrist supporters will abstain and this is inconsistent with equilibrium. Hence, either $B>0$ and in this case there will not be abstention in equilibrium, or it is exactly equal to zero. In this latter case there will be abstention of a subset of centrist voters, and $n_{1} \Delta_{1}=n_{3} \Delta_{2}$ can be rewritten as $\tilde{x}_{12} \Delta_{1}=\left(1-\tilde{x}_{23}\right) \Delta_{2}$ and it is verified in the case of a locationsymmetric equilibrium.

Proof of Proposition 7. First note that for small cost of voting $\tau$, either condition (i) or (ii) of Proposition 6 will hold generically. Formally, for any population size $N$ and party configuration $\mathcal{D} \equiv\left\{x_{1}, \Delta_{1}, \Delta_{2}\right\}$, there exists a $\bar{\tau}(N, \mathcal{D})>0$ such that for any two $\tau, \tau^{\prime} \leq \bar{\tau}(N, \mathcal{D}),\left[n_{1}(\tau, N) \Delta_{1}-n_{3}(\tau, N) \Delta_{2}\right] \times\left[n_{1}\left(\tau^{\prime}, N\right) \Delta_{1}-n_{3}\left(\tau^{\prime}, N\right) \Delta_{2}\right]>0$, where $n_{j}(\cdot)$ denotes the number of individuals voting for party $j$ in equilibrium. Assume then that $\tau<\bar{\tau}(N, \mathcal{D})$, and without loss of generality, also that $n_{1}(\tau, N) \Delta_{1}-n_{3}(\tau, N) \Delta_{2}>0$. Note that Proposition 6 implies that in this case abstention occurs in an interval $\left(\tilde{x}_{12}^{\ell}, \tilde{x}_{12}^{r}\right)$ around $\tilde{x}_{12}$. As a result, we can rewrite $n_{1}(\tau, N) \Delta_{1}>n_{3}(\tau, N) \Delta_{2}$ as $\tilde{x}_{12}^{\ell}(\tau, N) \Delta_{1}>$ $\left(1-\tilde{x}_{23}(\tau, N)\right) \Delta_{2}$, or simply as $\tilde{x}_{12}^{\ell} \Delta_{1}>\left(1-\tilde{x}_{23}\right) \Delta_{2}$.

Note that since $\tilde{x}_{12}^{\ell}\left(\tilde{x}_{12}^{r}\right)$ denotes the ideology of the voter who is indifferent between abstaining and voting for candidate 1 (2), it must be the case that $\tilde{x}_{12}^{\ell} \leq \tilde{x}_{12} \leq \tilde{x}_{12}^{r}$. In 
particular, $\tilde{x}_{12}^{\ell}$ and $\tilde{x}_{12}^{r}$ are given by the (unique) solution of system (22). ${ }^{15}$

$$
\left\{\begin{array}{c}
\frac{\left(1-\tilde{x}_{12}^{r}\right)\left(\tilde{x}_{12}-\tilde{x}_{12}^{l}\right) \Delta_{1}+\left(1-\tilde{x}_{23}\right)\left(\tilde{x}_{23}-\tilde{x}_{12}^{\ell}\right) \Delta_{2}}{N\left(1-\left(\tilde{x}_{12}^{r}-\tilde{x}_{12}^{\ell}\right)\right)\left(1-\left(\tilde{x}_{12}^{r}-\tilde{x}_{12}^{\ell}\right)-\frac{1}{N}\right)}=\frac{\tau}{2} \\
\frac{\tilde{x}_{12}^{\ell}\left(\tilde{x}_{12}^{r}-\tilde{x}_{12}\right) \Delta_{1}+\left(1-\tilde{x}_{23}\right)\left(\tilde{x}_{23}-\tilde{x}_{12}^{r}\right) \Delta_{2}}{N\left(1-\left(\tilde{x}_{12}^{r}-\tilde{x}_{12}^{l}\right)\right)\left(1-\left(\tilde{x}_{12}^{r}-\tilde{x}_{12}^{l}\right)-\frac{1}{N}\right)}=\frac{\tau}{2}
\end{array}\right.
$$

Assume from now on that voting is almost costless. Note that in this case $\tilde{x}_{12}^{\ell} \rightarrow \tilde{x}_{12}$ and $\tilde{x}_{12}^{r} \rightarrow \tilde{x}_{12}$, so that turnout $t=1-\left(\tilde{x}_{12}^{r}-\tilde{x}_{12}^{\ell}\right) \rightarrow 1$.

First, we show that turnout is decreasing in $\theta_{1}$. Note that $\tilde{x}_{12}^{\ell}<\tilde{x}_{12}$ if and only if $\tilde{x}_{12} \Delta_{1}>\left(1-\tilde{x}_{23}\right) \Delta_{2}$, which is implied by $\tilde{x}_{12}^{\ell} \Delta_{1}>\left(1-\tilde{x}_{23}\right) \Delta_{2}$. Evaluating the total differential of the two equations in system (22) with almost costless voting yields

$$
\begin{aligned}
\frac{\partial \tilde{x}_{12}^{r}}{\partial \theta_{1}} & =\frac{\tilde{x}_{12}\left(\Delta_{1}\left(1-\tilde{x}_{12}\right)+\left(1-\tilde{x}_{23}\right) \Delta_{2}\right)+\frac{\tau(2 N-1)}{2}}{\left(\left(1-\tilde{x}_{12}\right) \Delta_{1}+\left(1-\tilde{x}_{23}\right) \Delta_{2}\right)\left(\tilde{x}_{12} \Delta_{1}-\left(1-\tilde{x}_{23}\right) \Delta_{2}\right)+\frac{\tau(2 N-1)}{2} \Delta_{1}} \Delta_{1} \frac{\partial \tilde{x}_{12}}{\partial \theta_{1}}>0 \\
\frac{\partial \tilde{x}_{12}^{\ell}}{\partial \theta_{1}} & =\frac{\left(1-\tilde{x}_{12}\right)\left(\tilde{x}_{12} \Delta_{1}-\left(1-\tilde{x}_{23}\right) \Delta_{2}\right)+\frac{\tau(2 N-1)}{2}}{\left(\left(1-\tilde{x}_{12}\right) \Delta_{1}+\left(1-\tilde{x}_{23}\right) \Delta_{2}\right)\left(\tilde{x}_{12} \Delta_{1}-\left(1-\tilde{x}_{23}\right) \Delta_{2}\right)+\frac{\tau(2 N-1)}{2} \Delta_{1}} \Delta_{1} \frac{\partial \tilde{x}_{12}}{\partial \theta_{1}}>0,
\end{aligned}
$$

from where it follows that $\frac{\partial \tilde{x}_{12}^{r}}{\partial \theta_{1}}>\frac{\partial \tilde{x}_{12}}{\partial \theta_{1}}>\frac{\partial \tilde{x}_{12}^{\ell}}{\partial \theta_{1}}$. Using this we can show that campaign spending of party 1 in equilibrium must be higher with voluntary voting than with compulsory voting. Notice that

$$
\frac{\partial m_{1}(\theta, x, \tau)}{\partial \theta_{1}}=\frac{\partial \frac{\tilde{x}_{12}^{\ell}}{t}}{\partial \theta_{1}}=\frac{\frac{\partial \tilde{x}_{12}^{\ell}}{\partial \theta_{1}} t+\tilde{x}_{12}^{l}\left(\frac{\partial \tilde{x}_{12}^{r}}{\partial \theta_{1}}-\frac{\partial \tilde{x}_{12}^{\ell}}{\partial \theta_{1}}\right)}{t^{2}}
$$

and that

$$
\frac{\partial m_{1}(\theta, x, \tau)}{\partial \theta_{1}}>\frac{\partial \tilde{x}_{12}}{\partial \theta_{1}} \text { if and only if } \frac{\partial \tilde{x}_{12}^{\ell}}{\partial \theta_{1}} t+\tilde{x}_{12}^{l}\left(\frac{\partial \tilde{x}_{12}^{r}}{\partial \theta_{1}}-\frac{\partial \tilde{x}_{12}^{\ell}}{\partial \theta_{1}}\right)>t^{2} \frac{\partial \tilde{x}_{12}}{\partial \theta_{1}}
$$

which is always true in the case of almost costless voting. Since $\tilde{x}_{12}^{\ell} / t$ is the vote share of party 1 , it follows that the marginal return of campaign spending with voluntary voting

\footnotetext{
${ }^{15}$ Note that (22) admits a unique solution. By subtracting the second equation of $(22)$ from the first and substituting turnout $t=1-\left(\tilde{x}_{12}^{r}-\tilde{x}_{12}^{\ell}\right)$, we have that $\tilde{x}_{12}^{\ell}(t)=t \tilde{x}_{12}+(1-t)\left(1-\tilde{x}_{23}\right) \frac{\Delta_{2}}{\Delta_{1}}$, which is increasing in $t$ since $\tilde{x}_{12} \Delta_{1}>\tilde{x}_{12}^{\ell} \Delta_{1}>\left(1-\tilde{x}_{23}\right) \Delta_{2}$. Plugging this in the second equation of $(22)$ we get

$$
\tilde{x}_{12}^{r}(t)=\tilde{x}_{12}+\frac{\frac{\tau t(N t-1)}{2}-\left(1-\tilde{x}_{23}\right)\left(\tilde{x}_{23}-\tilde{x}_{12}\right) \Delta_{2}}{t\left(\tilde{x}_{12} \Delta_{1}-\left(1-\tilde{x}_{23}\right) \Delta_{2}\right)},
$$

which is also increasing in $t$. Hence, existence and uniqueness of a solution to the system (22) follows from the fact that $1-t-\tilde{x}_{12}^{r}(t)+\tilde{x}_{12}^{l}(t)=0$ has a unique solution.
} 
is higher than with compulsory voting, and therefore that campaign spending of party 1 in equilibrium must be higher with voluntary voting than with compulsory voting.

Proceeding in a similar way we can compare the marginal return of campaign spending when voting is voluntary and compulsory for parties 2 and 3. First note that

$$
\begin{gathered}
\frac{\partial \tilde{x}_{12}^{r}}{\partial \theta_{2}}=\frac{\left(1-2\left(\tilde{x}_{23}-\tilde{x}_{12}\right)\right)\left(\left(1-\tilde{x}_{12}\right) \Delta_{1}+\left(1-\tilde{x}_{23}\right) \Delta_{2}\right)+\frac{\tau(2 N-1)}{2}}{\left(\tilde{x}_{12} \Delta_{1}-\left(1-\tilde{x}_{23}\right) \Delta_{2}\right)\left(\left(1-\tilde{x}_{12}\right) \Delta_{1}+\left(1-\tilde{x}_{23}\right) \Delta_{2}\right)+\frac{\tau(2 N-1)}{2} \Delta_{1}} \frac{\partial \tilde{x}_{12}}{\partial \theta_{2}} \Delta_{1} \\
\frac{\partial \tilde{x}_{12}^{\ell}}{\partial \theta_{2}}=\frac{2\left(\tilde{x}_{23}-\tilde{x}_{12}\right)\left(\tilde{x}_{12} \Delta_{1}-\left(1-\tilde{x}_{23}\right) \Delta_{2}\right)+\frac{\tau(2 N-1)}{2}}{\left(\tilde{x}_{12} \Delta_{1}-\left(1-\tilde{x}_{23}\right) \Delta_{2}\right)\left(\left(1-\tilde{x}_{12}\right) \Delta_{1}+\left(1-\tilde{x}_{23}\right) \Delta_{2}\right)+\frac{\tau(2 N-1)}{2} \Delta_{1}} \frac{\partial \tilde{x}_{12}}{\partial \theta_{2}} \Delta_{1}<0,
\end{gathered}
$$

where we used the fact that

$$
\begin{gathered}
\tilde{x}_{12}=\frac{x_{1}+x_{2}}{2}+\alpha \frac{v\left(\theta_{1}\right)-v\left(\theta_{2}\right)}{\Delta_{1}}, \tilde{x}_{23}=\frac{x_{2}+x_{3}}{2}+\alpha \frac{v\left(\theta_{2}\right)-v\left(\theta_{3}\right)}{\Delta_{2}}, \text { and } \\
\frac{\partial \tilde{x}_{12}}{\partial \theta_{2}}=-\alpha \frac{v^{\prime}\left(\theta_{2}\right)}{\Delta_{1}}=-\frac{\Delta_{2}}{\Delta_{1}} \frac{\partial \tilde{x}_{23}}{\partial \theta_{2}} .
\end{gathered}
$$

Note then that $\frac{\partial m_{2}(\theta, x, \tau)}{\partial \theta_{2}}=\frac{\partial\left(\left(\tilde{x}_{23}-\tilde{x}_{12}^{r}\right) / t\right)}{\partial \theta_{2}}>\frac{\partial \tilde{x}_{23}}{\partial \theta_{2}}-\frac{\partial \tilde{x}_{12}}{\partial \theta_{2}}$ if and only if

$$
\frac{\partial \tilde{x}_{12}^{r}}{\partial \theta_{2}}+\left(\tilde{x}_{23}-\tilde{x}_{12}\right)\left(\frac{\partial \tilde{x}_{12}^{\ell}}{\partial \theta_{2}}-\frac{\partial \tilde{x}_{12}^{r}}{\partial \theta_{2}}\right)<\frac{\partial \tilde{x}_{12}}{\partial \theta_{2}}
$$

or equivalently,

$$
\Delta_{1}\left(\tilde{x}_{23}-\tilde{x}_{12}\right)>\left(1-\tilde{x}_{23}\right)\left(\Delta_{1}+\Delta_{2}\right)
$$

Proceeding similarly, for party 3 , we have that

$$
\frac{\partial m_{3}(\theta, x, \tau)}{\partial \theta_{3}}=\frac{\partial\left(\frac{1-\tilde{x}_{23}}{t}\right)}{\partial \theta_{3}}=-\frac{\partial \tilde{x}_{23}}{\partial \theta_{3}}-\left(1-\tilde{x}_{23}\right)\left(\frac{\partial \tilde{x}_{12}^{\ell}}{\partial \theta_{3}}-\frac{\partial \tilde{x}_{12}^{r}}{\partial \theta_{3}}\right)>-\frac{\partial \tilde{x}_{23}}{\partial \theta_{3}}
$$

if and only if

$$
\tilde{x}_{23}-\tilde{x}_{12}>1-\tilde{x}_{23}
$$

Note that (24) holds whenever (23) does. It follows that if (23) holds, the marginal return of campaign spending with voluntary voting is higher than with compulsory voting for all parties. This implies that when voting is voluntary, campaign spending of all parties must be higher in equilibrium than when voting is compulsory. 
Next, note that since $\theta_{2}>\theta_{k}$ for $k=1,3$ in any three party equilibrium with compulsory voting, then in equilibrium $\tilde{x}_{23}(\theta)>\bar{x}_{23} \equiv \frac{x_{2}+x_{3}}{2}$, and $\tilde{x}_{12}(\theta)<\bar{x}_{12} \equiv \frac{x_{1}+x_{2}}{2}$. Thus to establish that (23) holds it is enough to show that

$$
\Delta_{1}\left(\bar{x}_{23}-\bar{x}_{12}\right)>\left(1-\bar{x}_{23}\right)\left(\Delta_{1}+\Delta_{2}\right),
$$

or substituting,

$$
\left(x_{2}-\frac{1}{2}\right)+\left(\frac{x_{3}-x_{1}}{2}\right)>\frac{1}{2} .
$$

Thus when asymmetry $\left(x_{2}-\frac{1}{2}\right)$ and polarization $\left(\frac{x_{3}-x_{1}}{2}\right)$ are sufficiently high, all parties spend more in campaigning when voting is voluntary than when it is compulsory (note there exist multiple asymmetry/polarization configurations that satisfy this inequality).

\section{Appendix B}

Consider two parties $k$ and $j>k$ representing policy positions $x_{k}$ and $x_{j}>x_{k}$ and investing $\theta_{k}$ and $\theta_{j}$, respectively. Recall that there always exists a unique policy $\tilde{x}_{k j}$ such that a voter $i$ with ideal policy $z^{i}=\tilde{x}_{k j}$ would be completely indifferent between parties $k$ and $j$,

$$
\tilde{x}_{k j}=\frac{x_{k}+x_{j}}{2}+\alpha \frac{v\left(\theta_{k}\right)-v\left(\theta_{j}\right)}{\left|x_{j}-x_{k}\right|} .
$$

For $k<K$, let $r_{k}(\theta, x) \equiv \arg \min _{j>k}\left\{\tilde{x}_{k j}(\theta, x)\right\}$ denote the identity of $k$ 's relevant competitor to the right given $(x, \theta)$, and let $\tilde{r}_{k}(\theta, x) \equiv \min _{j>k}\left\{\tilde{x}_{k j}(\theta, x)\right\}$ denote the position of the voter that is indifferent between $k$ and $r_{k}(\theta, x)$. Similarly, for $k>1$, define $\ell_{k}(\theta, x) \equiv \arg \max _{j<k}\left\{\tilde{x}_{j k}(\theta, x)\right\}$ and $\tilde{\ell}_{k}(\theta, x) \equiv \max _{j<k}\left\{\tilde{x}_{j k}(\theta, x)\right\}$. For $k=1$, let $\tilde{\ell}_{k}(\theta, x) \equiv 0$, and for $k=K$, let $\tilde{r}_{k}(\theta, x) \equiv 1$. Let then $\Delta_{k}(\theta, x) \equiv x_{r(k)}-x_{\ell(k)}$ denote the distance between the policy positions represented by $r_{k}(\theta, x)$ and $\ell_{k}(\theta, x)$, and let $\delta_{k}^{r} \equiv\left(x_{r(k)}-x_{k}\right) / \Delta_{k}$. Then for any given $(\theta, x)$, as long as $0<\tilde{\ell}_{k}(\theta, x)<\tilde{r}_{k}(\theta, x)<1$, party $k$ 's vote share is given by

$$
m_{k}(\theta, x)=\tilde{r}_{k}(\theta, x)-\tilde{\ell}_{k}(\theta, x) .
$$

As we already mentioned before, the vote share $m_{k}$ as a function of $\theta_{k}$ will typically have one or more points of non-differentiability. Clearly, the first such point is at the value $\underline{\theta}_{k}\left(\theta_{-k}, x\right)$ for which $m_{k}(\theta, x)=0$ for $\theta_{k}<\underline{\theta}_{k}\left(\theta_{-k}, x\right)$ and $m_{k}(\theta, x)>0$ for $\theta_{k} \geq \underline{\theta}_{k}\left(\theta_{-k}, x\right)$; i.e., the minimum campaign investment at which $k$ obtains some votes. However, provided that $\theta_{k} \geq \underline{\theta}_{k}\left(\theta_{-k}, x\right)$ and given the identity of $k$ 's relevant competitors 
for $\left(x, \theta_{-k}\right)$, the vote share of an interior party $1<k<K$ is

$$
\begin{aligned}
m_{k}\left(\theta_{k} ; \theta_{-k}, x\right) & =\frac{\Delta_{k}}{2}+\frac{\alpha}{\Delta_{k}}\left(\frac{v\left(\theta_{k}\right)-v\left(\theta_{r(k)}\right)}{\delta_{k}^{r}}+\frac{v\left(\theta_{k}\right)-v\left(\theta_{\ell(k)}\right)}{\left(1-\delta_{k}^{r}\right)}\right) \\
& =\frac{\Delta_{k}}{2}+\frac{\alpha}{\Delta_{k}} \frac{v\left(\theta_{k}\right)-\left[\left(1-\delta_{k}^{r}\right) v\left(\theta_{r(k)}\right)+\delta_{k}^{r} v\left(\theta_{\ell(k)}\right)\right]}{\left(1-\delta_{k}^{r}\right) \delta_{k}^{r}}
\end{aligned}
$$

which is only a function of the distance between the policy represented by $k$ and that of its relevant neighbors, $\delta_{k}^{r} \Delta_{k}$ and $\delta_{k}^{\ell} \Delta_{k}$, and the campaign investment of $k$ and its relevant neighbors $\theta_{r(k)}$ and $\theta_{\ell(k)}$. Thus given the identity of $k$ 's relevant competitors for $\left(x, \theta_{-k}\right)$, the vote share mapping $m_{k}\left(\theta_{k} ; \theta_{-k}, x\right)$ is differentiable at $\theta_{k} \geq \underline{\theta}_{k}\left(\theta_{-k}, x\right)$, and the marginal vote share is given by

$$
\frac{\partial m_{k}}{\partial \theta_{k}}=\alpha v^{\prime}\left(\theta_{k}\right)\left(\frac{1}{\Delta_{k}^{r}}+\frac{1}{\Delta_{k}^{\ell}}\right)=\frac{\alpha}{\delta_{k}^{r}\left(1-\delta_{k}^{r}\right) \Delta_{k}^{T}} v^{\prime}\left(\theta_{k}\right)
$$

In particular, the marginal impact of campaigning on vote share given the identity of $k$ 's relevant competitors is well defined, and increases the larger is $\alpha$, the smaller is $\Delta_{k}$ and - given $\Delta_{k}$ - the larger is $\left|\delta_{k}^{r}-1 / 2\right|$. Generically, however, $m_{k}\left(\theta_{k} ; \theta_{-k}, x\right)$ will not be differentiable at all $\left(\theta_{k} ; \theta_{-k}, x\right)$. To see why this is the case note that

$$
\frac{\partial \tilde{x}_{k n}}{\partial \theta_{k}}=\frac{\alpha}{x_{n}-x_{k}} v^{\prime}\left(\theta_{k}\right)>\frac{\alpha}{x_{m}-x_{k}} v^{\prime}\left(\theta_{k}\right)=\frac{\partial \tilde{x}_{k m}}{\partial \theta_{k}}
$$

whenever $x_{m}>x_{n}$. Since parties $k$ and $n$ are closer substitutes for voters than parties $k$ and $m$, an increase in $\theta_{k}$ has a larger impact in how the electorate divides among $k$ and $n$ than in how the electorate divides between $k$ and $m$. We have then two possibilities. If $k$ 's relevant competitor at $\theta_{k}$ is $m$, then $n$ will not be the relevant competitor at $\theta_{k}^{\prime}>\theta_{k}$, and in this case there are no discontinuities in the marginal vote share. But if $n$ is $k$ 's relevant competitor at $\theta_{k}$, then it is possible that for sufficiently high $\theta_{k}^{\prime}, m$ becomes $k$ 's relevant competitor, "squeezing" $n$. In this case, the change in the identity of the relevant competitor $r_{k}(\theta, x)$ forces an (upward) jump in $\Delta_{k}^{r}(\theta, x) \equiv x_{r(k)}-x_{k}$, and therefore a downward jump in $\partial m_{k} / \partial \theta_{k}$ (see Figure 1 below).

It is apparent from Figure 1 that relying on the first order condition and a (local) second order condition can potentially be very misleading. To see this, consider $k$ 's best response from this first order approach, which is given by

$$
\theta_{k}=\Psi^{-1}\left(\frac{\delta_{k}^{r}\left(1-\delta_{k}^{r}\right) \Delta_{k}^{T}}{\alpha}\right)
$$

Now suppose that $x_{k} \rightarrow x_{k+1}$. Then $\delta_{k}^{r} \rightarrow 0$, and (28) implies that, unless the cost of campaigning increases very sharply, $\theta_{k}$ will eventually hit its upper bound. This logic, however, is not necessarily correct. While $k$ and $k+1$ are close substitutes, and therefore voters who rank $k$ and $k+1$ highest are very sensitive to differences in campaigning among these candidates, the "local market" can very well be small. In this case, while $k$ 's marginal vote share can be very high for a small interval of $\theta_{k}$, it will then drops to a much 

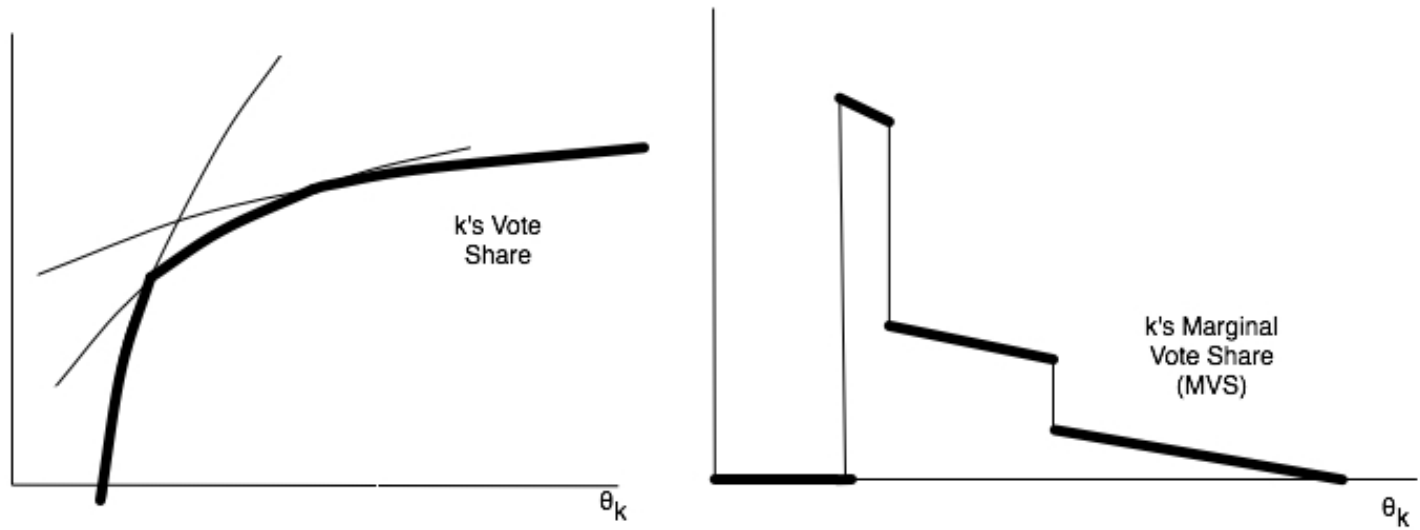

Figure 1: k's Vote Share and Marginal Vote Share.

smaller level as soon as $k$ 's relevant competitor changes from $k+1$ to the more distant $k+2$, a much worst substitute to $k$ in the eyes of voters. This is illustrated in Figure 2 , which shows that in this example the second discontinuity of the marginal vote share function would hit earlier than the intersection with the marginal cost schedule. Hence, the intersection of marginal cost and marginal vote share would be at a lower $\theta_{k}$ than in the absence of discontinuities. Under some conditions, however, the action identified as

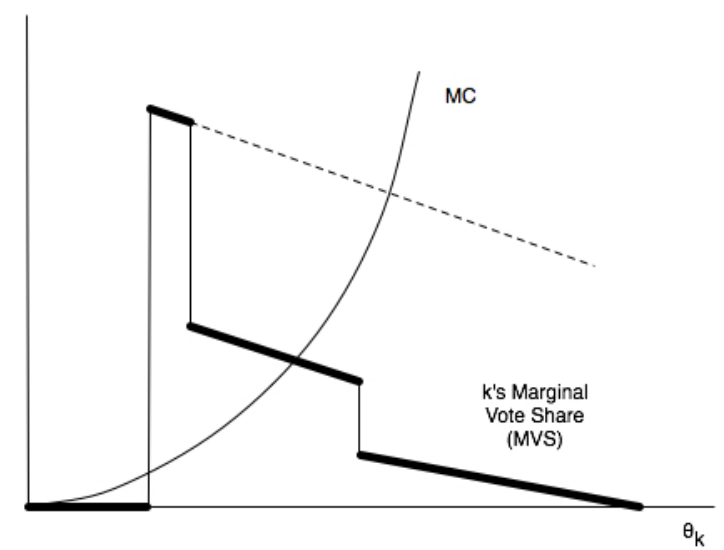

Figure 2: k's Marginal Vote Share and Marginal Cost of Campaigning (MC).

optimal by the first order condition will indeed be a best response. Consider for example the case of interior equilibria with two parties running for office (Proposition 1). In this case the identity of the relevant competitor is fixed by construction and therefore for any given $\theta_{2}$, 1's vote share mapping $m_{1}\left(\theta_{1} ; \theta_{2}, x\right)$ has two kinks, one at $\underline{t}$ such that $m_{1}\left(\underline{t} ; \theta_{2}, x\right) \equiv 0$ and one at $\bar{t}$ such that $m_{1}\left(\bar{t} ; \theta_{2}, x\right) \equiv 1$. In fact $\underline{t}=v^{-1}\left(v\left(\theta_{2}\right)-\Delta^{2} / \alpha\right)<$ $\theta_{2}$ and $\bar{t}=v^{-1}\left(v\left(\theta_{2}\right)+\Delta(1-\Delta) / \alpha\right)>\theta_{2}$. Thus marginal rent is well defined, continuous and decreasing at all points $\theta_{1} \in(\underline{t}, \bar{t})$. Since the first order conditions for 1 and 2 imply $\theta_{1}^{*}=\theta_{2}^{*}=\theta^{*}$, the kinks are not relevant. The same result holds for all location-symmetric (LS) electoral equilibria as we show in Lemma 1. 


\section{References}

Alesina, A. (1988): "Credibility and Policy Convergence in a Two-Party System with Rational Voters," American Economic Review, 78, 796-805.

Aragones, E., And T. R. Palfrey (2002): "Mixed Equilibrium in a Downsian Model with a Favored Candidate," Journal of Economic Theory, 103, 131-161.

Ashworth, S., And E. Bueno de Mesquita (2007): "Valence Competition and Platform Divergence," Typescript.

Austen-Smith, D. (1987): "Interest Groups, Campaign Contributions, and Probabilistic Voting," Public Choice, 54, 123-139.

- (2000): "Redistributing Income under Proportional Representation," Journal of Political Economy, 108(6), 1235-1269.

Austen-Smith, D., And J. S. Banks (1988): "Elections, Coalitions, and Legislative Outcomes," American Political Science Review, 82, 405-422.

Baron, D. P., And D. Diermeier (2001): "Elections, Governmens and Parliaments in Proportional Representation Systems," The Quarterly Journal of Economics, pp. 933-967.

Bartels, L. (1986): "Issue Voting under Uncertainty: An Empirical Test," American Journal of Political Science, 30, 709-728.

Baye, M., D. Kovenock, And C. De VRies (1994): "The Solution to the Tullock RentSeeking Game when R > 2: Mixed-strategy Equilibria and Mean Dissipation Rates," Public Choice, 81, 363-380.

- (1996): "The All-pay Auction with Complete Information," Economic Theory, 8, 291-305.

Berndardt, D., O. Camara, and F. Squintani (2009): "Competence and Ideology," Typeset, Marshall School of Business, USC.

Besley, T., And S. Coate (1997): "An Economic Model of Representative Democracy," The Quarterly Journal of Economics, 112(1), 85-114.

Börgers, T. (2004): "Costly Voting," American Economic Review, 94, 57-66.

Callander, S. (2008): "Political Motivations," Review of Economic Studies, 75, 671-697.

Carrillo, J. D., and M. Castanheira (2006): "Information and Strategic Polarization," Department of Economics, USC.

Coleman, J. J., And P. F. Manna (2000): "Congressional Campaign Spending and the Quality of Democracy," Journal of Politics, 62, 757-789.

Degan, A. (2008): "Mobilization and Persuasion of Political Advertising," Working Paper, UQAM. 
Eyster, E., And T. Kittsteiner (2007): "Party Platforms in Electoral Competition with Heterogeneous Constituencies," Theoretical Economics, 2, 41-70.

Feddersen, T. (1992): "A Voting Model Implying Duverger's Law and Positive Turnout," American Journal of Political Science, pp. 938-962.

Feddersen, T., I. Sened, And S. Wright (1990): "Rational Voting and Candidate Entry under Plurality Rule," American Journal of Political Science, 34, 1005-1016.

Gerber, A. S. (1998): "Estimating the Effect of Campaign Spending on Senate Election Outcomes Using Instrumental Variables," American Political Science Review, 134, 401-411.

Gerber, A. S., And D. P. Green (2000): "The Effect of Non-partisan Get-out-to-vote Drive: An Experimental Study of Leaftletting," Journal of Politics, 62, 846-857.

Green, D. P., and J. S. Krasno (1988): "Salvation for the Spendthrift Incumbent: Reestimating the Effects of Campaign Spending in House Elections," American Journal of Political Science, 32, 884-907.

Groseclose, T. (2001): "A Model of Candidate Location when one Candidate has a Valence Advantage," American Journal of Political Science, 45, 862-886.

Grossman, G. M., and E. Helpman (1996): "Electoral Competition and Special Interest Politics," The Review of Economic Studies, 63(2), 265-286.

Herrera, H., D. K. Levine, and C. Martinelli (2008): "Policy Platforms, Campaign Spending and Voter Participation," Journal of Public Economics, 92, 501-513.

Hotelling, H. (1929): "Stability in Competition," The Economic Journal, 39, 41-57.

IARYCZOwer, M., AND A. Mattozzi (2009): "On the Nature of Competition in Alternative Electoral Systems," HSS, California Institute of Technology.

Kenny, C., And M. McBurnett (1994): "An Individual-Level Multiequation Model of Expenditure Effects in Contested House Elections," American Political Science Review, 88(3), 699-707.

Krasa, S., And M. Polborn (2009): "Is Mandatory Voting Better than Voluntary Voting?," Games and Economic Behavior, 66, 275-291.

Krishna, V., and J. Morgan (2009): "Voluntary Voting: Costs and Benefits," typescript.

Ledyard, J. (1984): "The Pure Theory of Large two-candidate Elections," Public Choice, 44, $7-41$.

Lizzeri, A., And N. Persico (2001): "The Provision of Public Goods under Alternative Electoral Incentives," American Economic Review, 91, 225-239.

Meirowitz, A. (2007): "Electoral Contests, Incumbency Advantages and Campaign Finance," Princeton University.

Myerson, R. B. (1993): "Effectiveness of Electoral Systems for Reducing Government Corruption: A Game-Theoretic Analysis," Games and Economic Behavior, 5, 118-132. 
Osborne, M. J., And A. Slivinski (1996): "A Model of Political Competition with CitizenCandidates," The Quarterly Journal of Economics, 111(1), 65-96.

Palfrey, T., And H. Rosenthal (1983): "A Strategic Calculus of Voting," Public Choice, $41,7-53$.

(1985): "Voter Participation and Strategic Uncertainty," American Political Science Review, 79, 62-78.

PAlfrey, T. R. (1984): "Spatial Equilibrium with Entry," Review of Economic Studies, 51(1), $139-156$.

PAlfrey, T. R. (1989): "A Mathematical Proof of Duverger's Law," in Ordeshook, Peter C., Models of Strategic Choice in Politics, University of Michigan Press, Ann Arbor, 1989, 69-91.

Persico, N., and N. Sahuguet (2006): "Campaign Spending Regulation in a Model of Redistributive Politics," Economic Theory, 28(1), 95-124.

Persson, T., G. Roland, And G. Tabellini (2003): "How do Electoral Rules Shape Party Structures, Government Coalitions and Economic Policies," Department of Economics, UC Berkeley.

Persson, T., G. Tabellini, and F. Trebbi (2006): "Electoral Rules and Corruption," Journal of the European Economic Association, 1, 958-989.

Prat, A. (2002): "Campaign spending with office-seeking politicians, rational voters, and multiple lobbies," Journal of Economic Theory.

RekKas, M. (2007): "The Impact of Campaign Spending on Votes in Multiparty Elections," Review of Economics and Statistics, 89, 573-585.

Stokes, D. E. (1963): "Spatial Models of Party Competition," American Political Science Review, 57, 368-377.

Stratmann, T. (2009): "How prices matter in politics: The returns to campaign advertising," Public Choice.

Strom, K. (1990): "A Behavioral Theory of Competitive Political Parties," American Journal of Political Science, 34, 565-598.

TAYlor, C., AND H. Yildirim (2009): "Public Information and Electoral Bias," Forthcoming Games and Economic Behavior. 